\title{
Seroprevalence of hepatitis B and C in Nepal: a systematic review (1973-2017)
}

\author{
Marcelo Contardo Moscoso Naveira ${ }^{*}$ D, Komal Badal', Jagadish Dhakal, Neichu Angami Mayer, Bina Pokharel \\ and Ruben Frank Del Prado
}

\begin{abstract}
Introduction: Hepatitis B and C represent an important co-infection for people living with HIV worldwide. Nepal wants to be part of the international mobilization for viral hepatitis elimination, and has pursued better understanding of the epidemic in its territory through scientific research.

Methods: We performed a systematic review of seroprevalence studies hepatitis B and C in Nepal following the PRISMA 2009 Flow Diagram.

Results: Fifty-four scientific publications and reports were selected for this review. Nearly a quarter of these documents have been issued in recent years and many are authored by non-governmental organizations in Nepal. The collective of information displays a wide range of alarming prevalence rates, particularly for girls and women survivors of human trafficking and a progressive participation of civil society in viral hepatitis epidemiology research in the country.

Conclusion: This paper presents a most complete review of hepatitis B and C and HIV co-infection prevalence studies in different population groups from 1973 to 2016. A comprehensive analysis of the epidemiology and apparent trends in public health research and policy making in Nepal are also addressed in this document. We expect this to be a most important tool for improvements in future interventions for both epidemics in the country.
\end{abstract}

Keywords: Viral hepatitis, Hepatitis B, Hepatitis C, Systematic review, Epidemiology, Nepal

\section{Background}

Viral hepatitis has become a leading cause of death and disability worldwide - estimated to be responsible for over 1.4 million deaths every year. Chronic viral hepatitis, mostly represented by the hepatitis $\mathrm{B}$ and $\mathrm{C}$ viruses infections (HBV and $\mathrm{HCV}$, respectively), is a major cause of increasing events of high morbidity and mortality such as cirrhosis, end-stage liver disease and hepatocellular carcinoma. Both viruses are more easily transmissible than HIV [1].

The Sustainable Development Goals (SDG) establishes the year of 2030 as a desirable deadline for the end of many epidemics, including viral hepatitis. Nepal, a landlocked central Himalayan country in South Asia, has committed to the seventeen ambitious goals of SDG, and pursues to graduate from the least developed country rank by 2022 .

\footnotetext{
* Correspondence: mnaveir1@alumni.jh.edu; talktobadal@gmail.com; badalk@unaids.org

UNAIDS Nepal, UN House, Pulchowk, Lalitpur GPO 107, Nepal
}

Nepal already presented remarkable achievements in infectious diseases, particularly the HIV response [2-4]. However, the understanding of viral hepatitis impacts to the country is limited. There is no national plan devised for the elimination of viral hepatitis and hepatitis $\mathrm{C}$ has only been briefly mentioned in the National HIV Strategic Plan 20162021 (Nepal HIVision 2020) [5].

Multiple community- and facility-based seroepidemiological surveys for viral hepatitis and co-infections have taken place in Nepal since 1973 [6-8]. Studies have assessed different population groups mostly in urban areas of Kathmandu Valley and to a lesser extent in other development regions.

As new National Guidelines for Viral Hepatitis are being developed, the Joint United Nations Programme on HIV/ AIDS in Nepal (UNAIDS Nepal) understands this comprehensive review is a most welcome tool for future research. We expect this document to be useful for mathematical

(C) The Author(s). 2018 Open Access This article is distributed under the terms of the Creative Commons Attribution 4.0 International License (http://creativecommons.org/licenses/by/4.0/), which permits unrestricted use, distribution, and 
models, advocacy for key populations, improvements in public health policy, and setting priorities for successful elimination of hepatitis B and C.

\section{Methods}

We conducted a systematic review of seroprevalence studies of hepatitis B and C following the PRISMA 2009 Flow Diagram [9]. Our main sources of data for this research were: 1) PubMed (Medline), through the following search expression "(("Hepatitis B"[Mesh] OR "Hepatitis B, Chronic"[Mesh] OR "Hepatitis B virus"[Mesh] OR "Hepatitis B Surface Antigens"[Mesh] OR "Hepatitis B Antibodies"[Mesh] OR "Hepatitis B"[Text] OR "Hepatitis C"[Mesh] OR "Hepatitis C, Chronic"[Mesh] OR "Hepatitis C Antibodies"[Mesh] OR "Hepatitis C"[Text]) AND ("Nepal”[Mesh] OR "Nepal"[Text]))"; 2) reports provided by the Government of Nepal (GoN); 3) reports authored by international agencies and non-governamental organizations (NGOs); and 4) personal correspondence to authors.

\section{Study selection}

Two researchers took part in all steps of the reviewing process. We assessed our initial search results for eligibility through title, abstract and full-text analysis. Duplicates were not identified, but two publications were found to be supplemental to previously evaluated studies. One review obtained during the search presented additional data for three studies unavailable in digital media. Personal correspondences were sent to authors to obtain additional information. We could not identify any repetition of datasets.

Publications were considered eligible for inclusion if they presented own and original data (absolute numbers or percentage) for any population group, Nepalese or residing in Nepal, at any given site and time for at least one of the following outcomes of interest: 1) hepatitis B seroprevalence, active infection or exposure; and 2) hepatitis $C$ seroprevalence as detected by anti-HCV tests.

Selected publications were excluded if full-text material could not be retrieved, if published before 1981 and if abstract could not provide sufficient information for any of the three outcomes of interest.

\section{Data extraction}

The following data were then extracted from each eligible study included in this review: year of publication, population group, site, month and/or year of data collection, sample size, numbers/percentage of positive results for hepatitis B, C, HIV and syphilis; and authors' name.

Seven studies did not provide details of which tests were used to define active HBV infection [7, 10-15]. One study identified did not provide details about which tests were used to define seroprevalence of exposition to HBV [16].
We chose to display results for every study total population and for as many subgroups as possible. Figures were obtained through full-text analysis and personal correspondence with authors. All numbers were thoroughly revised.

\section{Results}

This review selection process is depicted in Fig. 1, as adapted from the PRISMA 2009 Flow Diagram [9]. Initial search expression resulted in ninety different records with no duplicates. Forty-two citations were excluded after title, abstract and full-text screening and one citation was found to be supplemental. One report from Asian Network of People Who Use Drugs (ANPUD), one from HEPA Foundation/United Nations Office on Drugs and Crime (UNODC), one from United Nations Development Program (UNPD), two from Nepal Red Cross Society (NRCS) and six from Ministry of Health of Nepal $(\mathrm{MoH})$, including the Global AIDS Response Progress Report 2015 (GARRP), were added to the group of forty-eight eligible citations, resulting in fifty-nine documents.

Five records were excluded because full-text could not be retrieved and their abstracts did not have data on any of the three outcomes of interest. Three additional documents from 1987 to 1990, which were not listed in PubMed, were identified from a review and later included in the collective. Fifty-seven documents relevant to fifty-five relevant prevalence studies were selected for this review.

Table 1 presents the collective of viral hepatitis prevalence studies with stratified population groups according to WHO key terms.

Table 2 presents an analysis of the reviewed data and Cochran's Q tests performed by Weill Cornell Medical College in Qatar. Estimated prevalence and heterogeneity has been presented for five population groups: PWID, populations at intermediate risk, populations at low risk (general population), populations with liver-related conditions and special clinical populations [17].

\section{Population groups}

Candidates for blood donation account for just seven prevalence studies and yet represent approximately $90 \%$ of the population evaluated for viral hepatitis in Nepal since 1973. This overwhelming presence of candidates for blood donation in seroprevalence studies does not contribute to the understanding of populations at increased risk of $\mathrm{HBV}, \mathrm{HCV}$ and HIV co-infection in Nepal as they rarely present seroprevalence rates higher than $1 \%[12,13,18-22]$.

It is understood that people at increased risk of HBV, $\mathrm{HCV}$ and HIV co-infections should be properly represented in our review. We have succeeded to identify studies for most groups of interest: general population, children, adults, pregnant women, people who inject drugs 


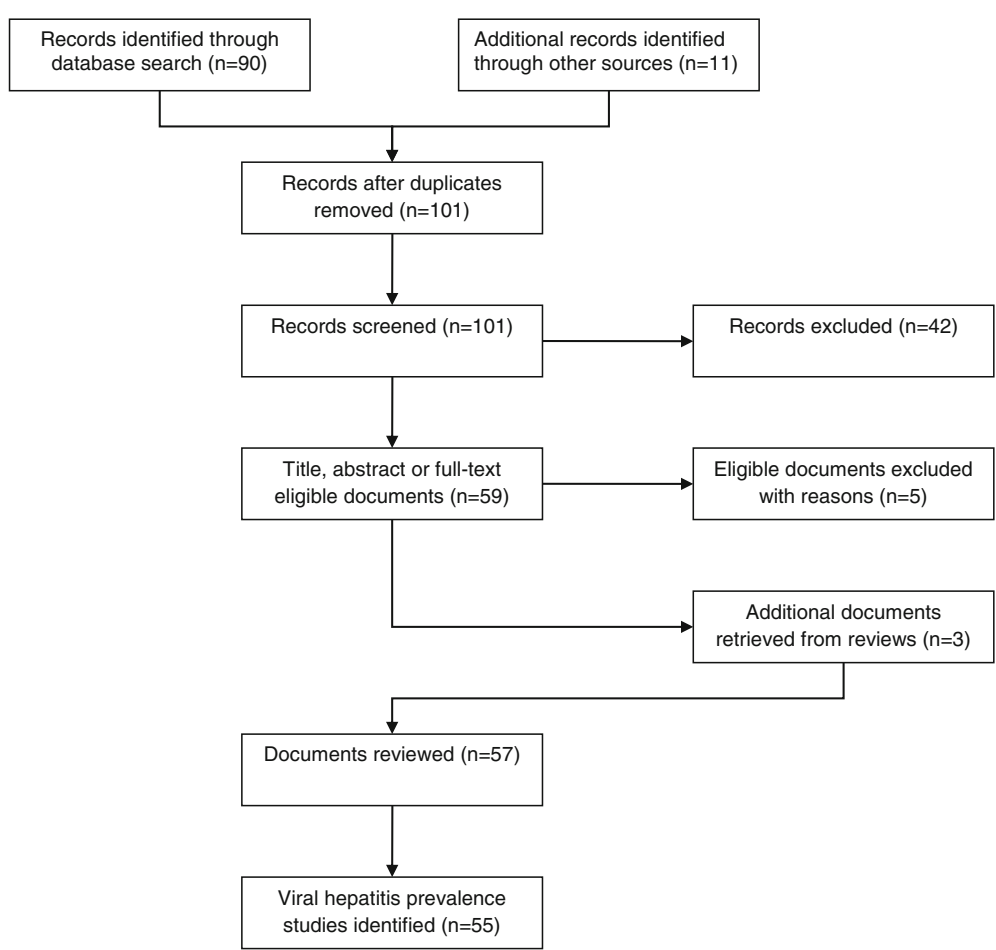

Fig. 1 Flow of article selection for the viral hepatitis B and C prevalence studies in Nepal

(PWID), patients attending healthcare services, sexual and household contacts of people chronically infected by HBV, sex workers $(\mathrm{SW})$, healthcare workers $(\mathrm{HCW})$, migrant workers, refugees/displaced persons and survivors of human trafficking.

We found only one survey of viral hepatitis in lesbian, gay, bisexual or transgender population (LGBT), including men who have sex with other men (MSM); and another in people with history of incarceration. All collected documents only referred to drug use as injection and did not acknowledge people who use drugs (PWUD) or different methods of drug administration (e.g. smoking heroin). [23, 24].

\section{Hepatitis B}

A disease preventable by vaccination, hepatitis $B$ has been identified in our review in forty-six studies. HBsAg (surface antigen) positive tests had highest values in PWID (1.3-81.9\%), [8, 25] patients with jaundice, chronic liver disease, cirrhosis or hepatocellular carcinoma (7.5-60\%); $[16,26-28]$ sexual and household contacts of people chronically infected by HBV (6.6-31), [29] girls and women survivors of sex trafficking (30\%), [7] Tibetan population living in Kathmandu Valley (10-20\%) [30] and Nepalese people outside Nepal (2.7-19.3) [31, 32]. On the other hand, the overall prevalence of hepatitis B in Nepal is estimated at $0.9 \%$, [33].

Children, adults and general population cohorts also present interesting ranges for figures of hepatitis
B seroprevalence. Older age groups present higher values for HBsAg [20,34] and children born after vaccine implementation display reduced disease prevalence. [35].

Unfortunately, only one document presents viral hepatitis prevalence in LGBT population (MSM included), but it lacks important information on sample size and number of positive tests.

Exposure to hepatitis B virus, defined by anti-HBc (antibody against core antigen), has been assessed only in one key population - PWID, in two studies nearly twenty years apart. In 1996, more than $80 \%$ of PWID had positive results for anti-HBc [25] and in 2015, when less than $45 \%$ had positive results for the same marker [8].

\section{Hepatitis C}

One of the most important causes of morbidity [36] and mortality, particularly for people living with HIV (PLWH), [37] hepatitis $C$ seroprevalence has been featured in thirty-one studies, in a total of approximately one million people in Nepal. Prevalence rates range from zero to more than $80 \%$, with highest figures found in PWID (85.5 in males; 24.6 in females), [38] PLHIV (43.3) and patients with hepatocellular carcinoma (17.6). [27, 38, 39].

Regardless of key population status, different prevalence rates have been observed in males and females [40]. Statistically significant differences according to gender can be verified in studies by Shrestha AC et al., between male and female candidates for blood donation in 2009 (0.69 vs. 
Table 1 Studies reporting hepatitis B and C in Nepal

\begin{tabular}{|c|c|c|c|c|c|c|c|c|c|c|}
\hline \multirow[t]{2}{*}{ SN } & \multirow[t]{2}{*}{ YEAR } & \multirow[t]{2}{*}{ POPULATION, SITE, TIME } & \multirow{2}{*}{$\begin{array}{l}\text { SAMPLE SIZE, } \\
\mathrm{N}\end{array}$} & \multirow{2}{*}{$\begin{array}{l}\text { ANTI- } \\
\text { HIV(\%) }\end{array}$} & \multicolumn{3}{|l|}{ HBV } & \multirow{2}{*}{$\begin{array}{l}\text { ANTI- } \\
\text { HCV }\end{array}$} & \multirow[t]{2}{*}{ NOTES } & \multirow[t]{2}{*}{ AUTHOR(S) } \\
\hline & & & & & HBSAG(\%) & $\begin{array}{l}\text { ANTI- } \\
\text { HBC(\%) }\end{array}$ & $\begin{array}{l}\text { ANTI- } \\
\text { HBS(\%) }\end{array}$ & & & \\
\hline 1 & 1973 & $\begin{array}{l}\text { Patients attending hospitals in Kathmandu } \\
\text { during infectious hepatitis outbreak, January } \\
1973 \text { to October } 1973\end{array}$ & $\begin{array}{l}53 \text { sera } \\
\text { samples }\end{array}$ & - & $0(0.00)$ & - & - & - & - & $\begin{array}{l}\text { Hillis A et al. } \\
\text { [6] }\end{array}$ \\
\hline \multirow[t]{2}{*}{2} & 1984 & $\begin{array}{l}\text { Hospitalized patients with jaundice referred } \\
\text { to Shree Tribhubn Chandra Military Hospital, } \\
\text { Kathmandu and Infectious Disease Hospital, } \\
\text { Teku, } 1981 \text { to } 1982\end{array}$ & 41 patients & - & $6(7.50)$ & - & - & - & - & $\begin{array}{l}\text { Kane MA et } \\
\text { al. [28] }\end{array}$ \\
\hline & & $\begin{array}{l}\text { Outpatients referred to Shree Tribhubn } \\
\text { Chandra Military Hospital, Kathmandu and } \\
\text { Infectious Disease Hospital, Teku, } 1981 \text { to } \\
1982\end{array}$ & 39 patients & - & & - & - & - & - & \\
\hline \multirow[t]{7}{*}{3} & \multirow[t]{7}{*}{1987} & Children age 0-10 years, Surkhet Valley & 45 children $^{a}$ & - & (6.6) & - & $(22.2)$ & - & - & \multirow{7}{*}{$\begin{array}{l}\text { Shreshta } \\
\text { SM. [72] }\end{array}$} \\
\hline & & $\begin{array}{l}\text { Children, teenagers and adults age 11- } \\
20 \text { years, Surkhet Valley }\end{array}$ & $\begin{array}{l}65 \text { children, } \\
\text { teenager and } \\
\text { adults }^{a}\end{array}$ & - & $(3.0)$ & - & $(46.6)$ & - & - & \\
\hline & & Adults age $21-40$ years, Surkhet Valley & 82 adults $^{a}$ & - & $(9.7)$ & - & $(44.0)$ & - & - & \\
\hline & & Adults $41+$ years, Surkhet Valley & 33 adults $^{\mathrm{a}}$ & - & $(6.0)$ & - & $(25.0)$ & - & - & \\
\hline & & $\begin{array}{l}\text { Girls and women, } 0-41+\text { years, Surkhet } \\
\text { Valley }\end{array}$ & - & - & $(9.8)$ & & - & - & - & \\
\hline & & Boys and men, $0-41+$ years, Surkhet Valley & - & - & $(4.4)$ & - & - & - & - & \\
\hline & & $\begin{array}{l}\text { General population age } 0-41+\text {, Surkhet } \\
\text { Valley }\end{array}$ & 225 people $^{a}$ & - & $(6.6)$ & - & $(35.0)$ & - & - & \\
\hline \multirow[t]{12}{*}{4} & \multirow[t]{12}{*}{1987} & $\begin{array}{l}\text { Tibetan children age } 0-9 \text { years, Hemza, } \\
\text { Tashi Ling, Pokhara; Jawalkhel, Kathmandu } \\
\text { Valley }\end{array}$ & 34 children & - & $(20.0)$ & $(32.0)^{\mathrm{a}}$ & - & - & - & \multirow[t]{12}{*}{$\begin{array}{l}\text { Shrestha } \\
\text { SM. [30] }\end{array}$} \\
\hline & & $\begin{array}{l}\text { Tibetan children, teenagers and adults age } \\
\text { 10-19 years, Hemza, Tashi Ling, Pokhara; } \\
\text { Jawalkhel, Kathmandu Valley }\end{array}$ & $\begin{array}{l}79 \text { children, } \\
\text { teenagers and } \\
\text { adults }\end{array}$ & - & $(10.0)$ & $(39.0)^{\mathrm{a}}$ & - & - & - & \\
\hline & & $\begin{array}{l}\text { Tibetan adults age } 20-29 \text { years, Hemza, } \\
\text { Tashi Ling, Pokhara; Jawalkhel, Kathmandu } \\
\text { Valley }\end{array}$ & 106 adults & - & $(18.0)$ & $(55.0)^{\mathrm{a}}$ & - & - & - & \\
\hline & & $\begin{array}{l}\text { Tibetan adults age } 30-39 \text { years, Hemza, } \\
\text { Tashi Ling, Pokhara; Jawalkhel, Kathmandu } \\
\text { Valley }\end{array}$ & 61 adults & - & $(20.0)$ & $(46.0)^{a}$ & - & - & - & \\
\hline & & $\begin{array}{l}\text { Tibetans age } 40-49 \text { years, Hemza, Tashi } \\
\text { Ling, Pokhara; Jawalkhel, Kathmandu Valley }\end{array}$ & 95 adults & - & $(15.0)$ & $(36.0)^{a}$ & - & - & - & \\
\hline & & $\begin{array}{l}\text { Tibetans age 0-49 years, Hemza, Tashi Ling, } \\
\text { Pokhara; Jawalkhel, Kathmandu Valley }\end{array}$ & 375 people & - & $(16.0)$ & $(45.0)^{a}$ & - & - & - & \\
\hline & & $\begin{array}{l}\text { Nepalese children age } 0-9 \text { years, Hemza, } \\
\text { Tashi Ling, Pokhara; Jawalkhel, Kathmandu } \\
\text { Valley }\end{array}$ & 113 children & - & $(0.0)$ & $(3.5)^{\mathrm{a}}$ & - & - & - & \\
\hline & & $\begin{array}{l}\text { Nepalese children, teenagers and adults age } \\
\text { 10-19 years, Hemza, Tashi Ling, Pokhara; } \\
\text { Jawalkhel, Kathmandu Valley }\end{array}$ & $\begin{array}{l}198 \text { children, } \\
\text { teenagers and } \\
\text { adults }\end{array}$ & - & $(0.5)$ & $(5.0)^{\mathrm{a}}$ & - & - & - & \\
\hline & & $\begin{array}{l}\text { Nepalese adults age } 20-29 \text { years, Hemza, } \\
\text { Tashi Ling, Pokhara; Jawalkhel, Kathmandu } \\
\text { Valley }\end{array}$ & 110 adults & - & (1.8) & $(5.4)^{\mathrm{a}}$ & - & - & - & \\
\hline & & $\begin{array}{l}\text { Nepalese adults age } 30-39 \text { years, Hemza, } \\
\text { Tashi Ling, Pokhara; Jawalkhel, Kathmandu } \\
\text { Valley }\end{array}$ & 49 adults & - & $(0.0)$ & $(32.6)^{a}$ & - & - & - & \\
\hline & & $\begin{array}{l}\text { Nepalese adults age } 40-49 \text { years, Hemza, } \\
\text { Tashi Ling, Pokhara; Jawalkhel, Kathmandu } \\
\text { Valley }\end{array}$ & 25 adults & - & $(0.0)$ & $(16.0)^{a}$ & - & - & - & \\
\hline & & Nepalese adults age $50+$ years, Hemza, & 45 adults & - & $(2.2)$ & $(7.8)^{\mathrm{a}}$ & - & - & - & \\
\hline
\end{tabular}


Table 1 Studies reporting hepatitis B and C in Nepal (Continued)

SN YEAR POPULATION, SITE, TIME SAMPLE SIZE,

$\mathrm{N}$

\section{ANTI-}

HIV(\%)
HBV

HBSAG $(\%)$
Tashi Ling, Pokhara; Jawalkhel, Kathmandu

Nepalese age 11-41+ years, Hemza, Tashi Ling, Pokhara; Jawalkhel, Kathmandu Valley

51988 Patients during 1983 enterically transmitted non A-non B hepatitis outbreak, Kathmandu Valley

Controls during 1983 non A-non B hepatitis 100 people outbreak, Kathmandu Valley Kathmandu Valley

Breastfed infants age 2-6 months from six 26 infants villages of Kathmandu Valley

71989 Patients age 20-40 years attending four Kathmandu hospitals, Kathmandu, 1985

81990 Children age $0-5$ years

Children and teenagers age 6-15 years

Teenagers and adults age 16-41 years

Adults, $41+$ years

Girls and women, $0-41+$ years

Boys and men, $0-41+$ years

General population, $0-41+$ years

91991 Spouses of HBsAg chronic carriers

Offspring of HBsAg chronic carriers

Siblings of HBsAg chronic carriers

101993 Children age 0-4 years, Gurkha Contingent, Singapore

Children age 5-14 years, Gurkha

Contingent, Singapore

Children and adults age 15-24 years,

Gurkha Contingent, Singapore

Adults age 25-34 years, Gurkha Contingent, Singapore

Adults $35+$ years, Gurkha Contingent Singapore

Gurkha Community members, Gurkha Contingent, Singapore

111994 Patients with chronic hepatitis attending Bir Hospital, Kathmandu

\begin{tabular}{|c|c|c|c|c|c|c|c|}
\hline $\begin{array}{l}460 \text { sera } \\
\text { samples }\end{array}$ & $\begin{array}{l}0 \\
(0.00)\end{array}$ & $5(1.09)$ & $\begin{array}{l}64 \\
(13.91)\end{array}$ & - & - & - & $\begin{array}{l}\text { Mertens T } \\
\text { et al. [74] }\end{array}$ \\
\hline $\begin{array}{l}57 \text { healthy } \\
\text { children }\end{array}$ & - & $0(0.00)$ & - & $5(8.77)$ & - & - & $\begin{array}{l}\text { Shrestha SM } \\
{[75]}\end{array}$ \\
\hline $\begin{array}{l}359 \text { healthy } \\
\text { children ad } \\
\text { teenagers }\end{array}$ & - & $8(2.23)$ & - & $\begin{array}{l}15 \\
(4.18)\end{array}$ & - & - & \\
\hline $\begin{array}{l}1788 \text { healthy } \\
\text { teenagers and } \\
\text { adults }\end{array}$ & - & $16(0.89)$ & - & $\begin{array}{l}158 \\
(8.84)\end{array}$ & - & - & \\
\hline $\begin{array}{l}351 \text { healthy } \\
\text { adults }\end{array}$ & - & $2(0.57)$ & - & $\begin{array}{l}35 \\
(9.97)\end{array}$ & - & - & \\
\hline $\begin{array}{l}1529 \text { healthy } \\
\text { women }\end{array}$ & - & $9(0.59)$ & - & $\begin{array}{l}76 \\
(7.40)\end{array}$ & - & - & \\
\hline $\begin{array}{l}1026 \text { healthy } \\
\text { men }\end{array}$ & - & $17(1.65)$ & - & $\begin{array}{l}138 \\
(9.02)\end{array}$ & - & - & \\
\hline $\begin{array}{l}2555 \text { healthy } \\
\text { individuals }\end{array}$ & - & $26(1.02)$ & $\begin{array}{l}23 \\
(0.90)\end{array}$ & $\begin{array}{l}214 \\
(8.4)\end{array}$ & - & - & \\
\hline 34 people & - & $6(17.65)$ & - & $\begin{array}{l}15 \\
(44.12)\end{array}$ & - & - & $\begin{array}{l}\text { Shrestha SM } \\
\text { et al. [29] }\end{array}$ \\
\hline 73 people & - & $15(20.55)$ & - & $\begin{array}{l}17 \\
(23.29)\end{array}$ & - & - & \\
\hline 29 people & - & $9(31.03)$ & - & $\begin{array}{l}11 \\
(37.93)\end{array}$ & - & - & \\
\hline 177 children & - & 64 (19.28) & $\begin{array}{l}161 \\
(17.05)\end{array}$ & $\begin{array}{l}213 \\
(22.56)\end{array}$ & - & - & $\begin{array}{l}\text { Goh et al. } \\
\text { [32] }\end{array}$ \\
\hline
\end{tabular}

155 children

227 children and adults

289 people

96 people

944 people

$26(2.75)$

20 patients

Patients with cirrhosis attending Bir Hospital, 63 patients $(36.0)^{a}$

$34(13.60)^{a}$

Nuti M [10]

Reynolds

$\mathrm{RD}$ et al.

[73]

Mertens T et al. [74]

Shrestha SM [75]

[32]
Shreshta SM et al. [26] 
Table 1 Studies reporting hepatitis B and C in Nepal (Continued)

SN YEAR POPULATION, SITE, TIME SAMPLE SIZE,

$\mathrm{N}$

\section{ANTI- \\ HIV $(\%)$}

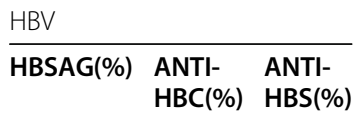

HBSAG(\%) ANTI- ANTI-

$\mathrm{HBC}(\%) \quad \mathrm{HBS}(\%)$

Kathmandu

Patients with hepatocellular carcinoma attending Bir Hospital, Kathmandu

Patients with chronic hepatitis, cirrhosis or hepatocellular carcinoma attending Bir Hospital, Kathmandu

Pregnant women attending Bir Hospital, Kathmandu

Medical or paramedical personnel serving at 296 people Bir Hospital, Kathmandu

121994 Female sex workers, Kathmandu Valley

131994 Villagers, Dharan, Sunsari; Pancha Kanya Village Development Committee, llam; Dhankuta Hile, Dhankuta; Basantapur Village Development Committee, Tehrathm

141995 Villagers, Bhadrakali and Kotyang villages, 1987

151996 People who inject drugs

161997 People who use drugs

People with chronic kidney disease

People with chronic liver disease

HBsAg carriers

Healthy individuals undergoing routine check-ups

171998 Patients age $\leq 15$ years attending Amp Pipal Hospital, Gorkha district, 1993

Patients age 16-25 years attending Amp Pipal Hospital, Gorkha district, 1993

Patients age 26-35 years attending Amp Pipal Hospital, Gorkha district, 1993

Patients age 36-45 years attending Amp Pipal Hospital, Gorkha district, 1993

Patients age 46-55 years attending Amp Pipal Hospital, Gorkha district, 1993

Patients age $\geq 56$ years attending Amp Pipal Hospital, Gorkha district, 1993

1999 Male villagers age $\leq 24$ years from Bhadrakali and Kotyang villages, August 1996 to September 1996

Female villagers age $\leq 24$ years from Bhadrakali and Kotyang villages, August 1996 to September 1996

Villagers age $\leq 24$ years from Bhadrakali and Kotyang villages, August 1996 to September 1996

Male villagers 25-34 years from Bhadrakali and Kotyang villages, August 1996 to

(14.28)

6

(3.38)

\begin{tabular}{|c|c|c|c|c|c|c|c|}
\hline 341 women & $\begin{array}{l}3 \\
(0.88)\end{array}$ & $37(10.85)^{a}$ & & & - & $\begin{array}{l}\text { Syphilis: } 73 \\
(21.41)\end{array}$ & $\begin{array}{l}\text { Bhatta P et } \\
\text { al. [76] }\end{array}$ \\
\hline $\begin{array}{l}303 \text { serum } \\
\text { samples }\end{array}$ & - & $0(0.00)$ & - & $6(1.98)$ & - & - & $\begin{array}{l}\text { Rai SK et al. } \\
{[77]}\end{array}$ \\
\hline $\begin{array}{l}676 \text { blood } \\
\text { samples }\end{array}$ & - & $2(0.29)$ & $\begin{array}{l}52 \\
(7.69)\end{array}$ & - & $\begin{array}{l}1 \\
(0.15)\end{array}$ & - & $\begin{array}{l}\text { Nakashima } \\
\text { K et al. [78] }\end{array}$ \\
\hline 72 people & - & $4(5.55)$ & $\begin{array}{l}59 \\
(81.94)\end{array}$ & - & $\begin{array}{l}58 \\
(80.55)\end{array}$ & - & $\begin{array}{l}\text { Shrestha SM } \\
\text { et al. [25] }\end{array}$ \\
\hline 72 people & - & $2(2.78)$ & \multicolumn{2}{|c|}{$44(61.11)^{b}$} & $\begin{array}{l}43 \\
(59,72)\end{array}$ & - & $\begin{array}{l}\text { Shrestha SM } \\
\text { et al. [16] }\end{array}$ \\
\hline 41 people & - & $1(2.44)$ & \multicolumn{2}{|c|}{$6(14.63)^{b}$} & $\begin{array}{l}1 \\
(2.44)\end{array}$ & - & \\
\hline 145 people & - & $57(39.31)$ & \multicolumn{2}{|c|}{$74(51.03)^{b}$} & $\begin{array}{l}12 \\
(8.27)\end{array}$ & - & \\
\hline 49 people & - & $49(100.00)$ & \multicolumn{2}{|c|}{$49(100.00)^{b}$} & $\begin{array}{l}0 \\
(0.00)\end{array}$ & - & \\
\hline 181 people & - & $9(4.97)$ & \multicolumn{2}{|c|}{$46(25.41)^{b}$} & $\begin{array}{l}0 \\
(0.00)\end{array}$ & - & \\
\hline \multirow[t]{6}{*}{$\begin{array}{l}101 \text { children } \\
\text { and adults }\end{array}$} & - & - & $\begin{array}{l}0 / 9 \\
(0.00)\end{array}$ & $\begin{array}{l}1 / 9 \\
(11.11)\end{array}$ & $\begin{array}{l}0 / 9 \\
(0.00)\end{array}$ & - & $\begin{array}{l}\text { De Bruyn et } \\
\text { al. [44] }\end{array}$ \\
\hline & - & - & $\begin{array}{l}0 / 34 \\
(0.00)\end{array}$ & $\begin{array}{l}0 / 34 \\
(0.00)\end{array}$ & $\begin{array}{l}4 / 34 \\
(11.76)\end{array}$ & - & \\
\hline & - & - & $\begin{array}{l}1 / 20 \\
(5.00)\end{array}$ & $\begin{array}{l}0 / 21 \\
(0.00)\end{array}$ & $\begin{array}{l}0 / 21 \\
(0.00)\end{array}$ & - & \\
\hline & - & - & $\begin{array}{l}2 / 14 \\
(14.28)\end{array}$ & $\begin{array}{l}2 / 13 \\
(15.38)\end{array}$ & $\begin{array}{l}0 / 13 \\
(0.00)\end{array}$ & - & \\
\hline & - & - & $\begin{array}{l}1 / 8 \\
(12.50)\end{array}$ & $\begin{array}{l}1 / 8 \\
(12.50)\end{array}$ & $\begin{array}{l}0 / 8 \\
(0.00)\end{array}$ & - & \\
\hline & - & - & $\begin{array}{l}3 / 10 \\
(30.00)\end{array}$ & $\begin{array}{l}4 / 10 \\
(40.00)\end{array}$ & $\begin{array}{l}0 / 10 \\
(0.00)\end{array}$ & - & \\
\hline $\begin{array}{l}44 \text { boys and } \\
\text { men }\end{array}$ & - & $0(0.00)$ & $1(2.27)$ & - & $\begin{array}{l}1 \\
(2.27)\end{array}$ & - & $\begin{array}{l}\text { Sawayama Y } \\
\text { et al. [40] }\end{array}$ \\
\hline $\begin{array}{l}43 \text { girls and } \\
\text { women }\end{array}$ & - & $1(2.32)$ & $1(2.32)$ & - & $\begin{array}{l}0 \\
(0.00)\end{array}$ & - & \\
\hline 87 people & - & $1(1.15)$ & $2(2.30)$ & - & $\begin{array}{l}1 \\
(1.15)\end{array}$ & - & \\
\hline 40 men & - & $0(0.00)$ & $2(5.00)$ & - & $\begin{array}{l}2 \\
(5.00)\end{array}$ & - & \\
\hline
\end{tabular}
Rai SK et al.

Nakashima Shrestha SM et al. [25] Shrestha SM t al. [16]

De Bruyn et

ANTI- NOTES AUTHOR(S) 
Table 1 Studies reporting hepatitis B and C in Nepal (Continued)

SN YEAR POPULATION, SITE, TIME SAMPLE SIZE,

N
ANTI-

HIV $(\%)$
HBV

HBSAG( $\%)$

\section{ANTI- NOTES \\ HCV \\ $\begin{array}{ll}\text { ANTI- } & \text { ANTI- } \\ \text { HBC(\%) } & \text { HBS(\%) }\end{array}$}

September 1996

Female villagers 25-34 years from Bhadrakali and Kotyang villages, August 1996 to September 1996

Villagers 25-34 years from Bhadrakali and Kotyang villages, August 1996 to September 1996

Male villagers 35-44 years from Bhadrakali and Kotyang villages, August 1996 to September 1996

Female villagers 35-44 years from Bhadrakali and Kotyang villages, August 1996 to September 1996

Villagers 35-44 years from Bhadrakali and Kotyang villages, August 1996 to September 1996

Male villagers 45-54 years from Bhadrakali and Kotyang villages, August 1996 to September 1996

Female villagers $45-54$ years from Bhadrakali and Kotyang villages, August 1996 to September 1996

Villagers 45-54 years from Bhadrakali and Kotyang villages, August 1996 to September 1996

Male villagers 55-64 years from Bhadrakali and Kotyang villages, August 1996 to September 1996

Female villagers 55-64 years from Bhadrakali and Kotyang villages, August 1996 to September 1996

Villagers 55-64 years from Bhadrakali and Kotyang villages, August 1996 to September 1996

Male villagers $\geq 65$ years from Bhadrakali and Kotyang villages, August 1996 to September 1996

Female villagers $\geq 65$ years from Bhadrakali and Kotyang villages, August 1996 to September 1996

Villagers $\geq 65$ years from Bhadrakali and Kotyang villages, August 1996 to September 1996

Female villagers age 15-90 years from Bhadrakali and Kotyang villages, August 1996 to September 1996

Male villagers age 15-90 years from Bhadrakali and Kotyang villages, August 1996 to September 1996

Villagers age 15-90 years from Bhadrakali and Kotyang villages, August 1996 to September 1996

192000 Healthy Nepalese Men from Eastern development region, October 1996 to March 1997

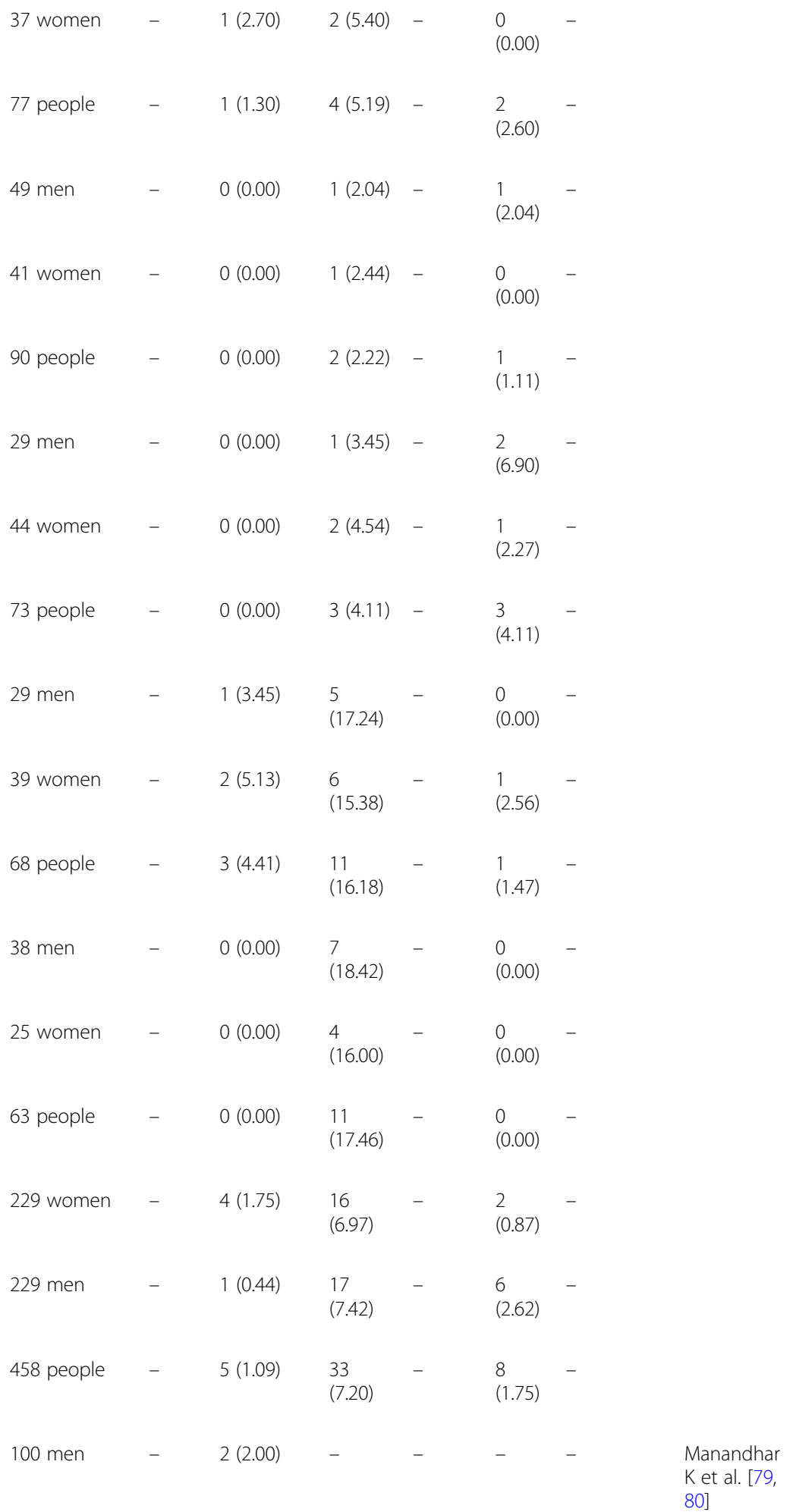


Table 1 Studies reporting hepatitis B and C in Nepal (Continued)

SN YEAR POPULATION, SITE, TIME SAMPLE SIZE,

$\mathrm{N}$

$\begin{array}{lll}100 \text { men } & - & 3(3.00) \\ 100 \text { men } & - & 4(4.00) \\ 97 \text { men } & - & 4(4.12)\end{array}$

Healthy Nepalese Men from Mid Western development region, October 1996 to March 1997

Healthy Nepalese Men from Far Western development region, October 1996 to March 1997

Healthy Nepalese Men from five different development regions, October 1996 to March 1997

202002 Nepalese men age 16-50 years who required medical check-ups for employment abroad, July 1999 to September 1999

212003 Boys and men < 19 years, Baba Medical Center, Kathmandu, September 2003 to June 2004

Men age 20-29 years, Baba Medical Center, Kathmandu, September 2003 to June 2004

Men age 30-39 years, Baba Medical Center, Kathmandu, September 2003 to June 2004

Men age 40+ years, Baba Medical Center, Kathmandu, September 2003 to June 2004

2003 People who inject drugs, Siddhi Polyclinic, Dillibazaar, Kathmandu

Adults without history of injection drug use, Siddhi Polyclinic, Dillibazaar, Kathmandu

232003 Candidates for blood donation at Blood Bank Centre, NRCS, Teaching Hospital, Bhairahawa, February 2001 to April 2003

242004 Sherpa people age 15-66 years, Lukla, Solukhumbu district, 2004

252005 Bhutanese refugees, Beldangi 2 Extension Camp, March 1998 to July 1998

262006 Healthcare workers of Bir Hospital, Kathmandu, December 2001 to February 2002

272006 Male villagers, migrant-returnes from India and non-migrants, from five village development committees, Doti District, April 2001

282007 Patients with liver cirrhosis or hepatocellular carcinoma attending Liver Foundation Nepal clinic, January 1998 to January 2004

Patients with liver cirrhosis attending Liver Foundation Nepal clinic, January 1998 to January 2004

Patients with hepatocellular carcinoma attending Liver Foundation Nepal clinic, January 1998 to January 2004

292008 Symptomatic people living with HIV/AIDS at Manipal Teaching Hospital, Pokhara, March

478 men

$18(3.76)$

2585 men

52 boys and

0

$0(0.00)$

men

(0.00)

375 men

5

(1.33)

$9(2.40)$

170 men

5

$7(4.11)$

(2.94)

30 men

$-$

1 (3.33)

400 people

$-$

400 people

1548 samples

(0.13)

$7(0.45)$

51 patients

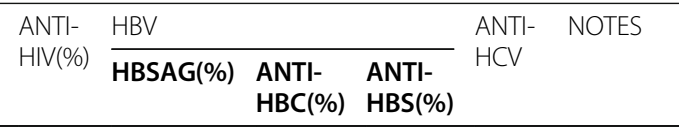

AUTHOR(S)

$\mathrm{HBC}(\%) \quad \mathrm{HBS}(\%)$

81 men $\quad-\quad 5(6.17)$

Bidya S [81]

103 people

$-\quad 2(1.94)$

25

(24.27)

$(22.33$

342

$17<40$ yrs

old -

467 people $\quad-\quad 4(0.86)$

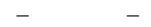

(85.5)

Shrestha IL

[38]

Joshi SK et al. [34]

Syphilis: 4/

545

145 people $\quad-\quad 2(1.38)$

21
$(14.48)$

3

(0.75)

2

(0.13)

Chander A

et al. [18]

Chiba $\mathrm{H}$ et

al. [82]

Shah BK et al. [83]

Shrestha SK et al. [68]

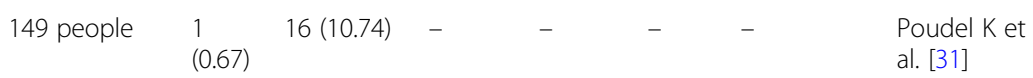

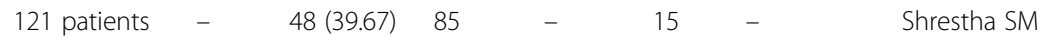
(70.25) (12.40) et al. [27]

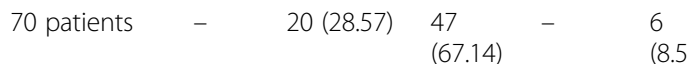

$28(54.90)$ 38

PLWH $1(1.85)^{\mathrm{a}}$ 
Table 1 Studies reporting hepatitis B and C in Nepal (Continued)

SN YEAR POPULATION, SITE, TIME SAMPLE SIZE,

$\mathrm{N}$

\section{ANTI-}

$H I V(\%)$

\section{HBV \\ HBSAG(\%) \\ ANTI- NOTES \\ $\mathrm{HCV}$ \\ $\mathrm{HBC}(\%) \quad \mathrm{HBS}(\%)$}

2004 to September 2005

30

\section{8}

Candidates for blood donation, NRSC, CBTS, hospital units/mobile camps all over Nepal, 2001 to 2002

Candidates for blood donation, NRSC, CBTS, 73,758 blood hospital units/mobile camps all over Nepal, samples 2002 to 2003

Candidates for blood donation, NRSC, CBTS, 76,647 blood hospital units/mobile camps all over Nepal, samples 2003 to 2004

Candidates for blood donation, NRSC, CBTS, 82,677 blood hospital units/mobile camps all over Nepal, samples 2004 to 2005

Candidates for blood donation, NRSC, CBTS, 103,067 blood hospital units/mobile camps all over Nepal, samples 2005 to 2006

Candidates for blood donation, NRSC, CBTS, 115,720 blood hospital units/mobile camps all over Nepal, samples 2006 to 2007

Candidates for blood donation, NRSC, CBTS, 524,328 blood hospital units/mobile camps all over Nepal, samples 2001 to 2007

312008 Sex trafficked women and girls assisted by Maiti Nepal, Kathmandu

246 women and girls 74

33,255

individual

samples Central Blood Transfusion Service, Nepa Red Cross Society (NRCS), Kathmandu, December 2006 to September 2007

People living with HIV/AIDS diagnosed during blood donation at the Central Blood Transfusion Service, NRCS, Kathmandu, December 2006 to September 2007

332009 Pregnant women admitted in the ward of NMCTH, Kathmandu, 2001 to 2007

342009 People who inject drugs on Oral Substitution Therapy (OST), Kathmandu Valley, June 2009

People who inject drugs, Kathmandu Valley, 82 people June 2009

2009 Male candidates for blood donation, NRCS, Central Blood Transfusion Service (CBTS) Kathmandu, March 2008 to September 2008

Female candidates for blood donation, NRCS, Central Blood Transfusion Service (CBTS), Kathmandu, March 2008 to September 2008

Candidates for blood donation age $\leq$ 20 years, NRCS, Central Blood Transfusion Service (CBTS), Kathmandu, March 2008 to September 2008

Candidates for blood donation age 3130 years, NRCS, Central Blood Transfusion Service (CBTS), Kathmandu, March 2008 to September 2008

Candidates for blood donation age 31-

65 individual

samples

tested positive

for anti-HIV

18,434 blood samples of

candidates

3282 blood samples of female candidates 3310 blood samples 9818 blood 12 samples

\begin{tabular}{|c|c|c|c|c|c|c|c|}
\hline 5602 & - & $18(0.32)^{a}$ & & & - & - & $\begin{array}{l}\text { Shreshta P } \\
\text { et al. [84] }\end{array}$ \\
\hline 118 people & - & - & - & - & $\begin{array}{l}95 \\
(80.50)\end{array}$ & - & $\begin{array}{l}\text { HEPA } \\
\text { Foundation } \\
\text { [85] }\end{array}$ \\
\hline
\end{tabular}
(30.08) $627(0.86)$ 384 (0.52) Karki S et al. [19] 417 (0.56) $911(1.23)$ $663(0.86)$ $(0.41)$

373

$887(0.86) \quad-\quad \ldots \quad-\quad 366$

$430(0.37)$

628

$4162(0.82)$

- Syphilis: Silverman J $48 \%$ et al. [7] Karki $S$ et al. [12]

7 (10.76)

47

(57.32)

25

$92(0.50)$

28

Coinfection Shrestha AC

(0.69) HIV/HCV: 8/ et al. [20] 128 $(0.06)$

$7(0.21)$ (0.33) $45(0.45)$

(0.12) 
Table 1 Studies reporting hepatitis B and C in Nepal (Continued)

$\mathrm{N}$

SN YEAR POPULATION, SITE, TIME SAMPLE SIZE,

\section{ANTI- \\ $H I V(\%)$}

\section{HBV}

HBSAG $\%$

\section{ANTI- NOTES \\ $\mathrm{HCV}$ \\ ANTI- ANTI-}

40 years, NRCS, Central Blood Transfusion Service (CBTS), Kathmandu, March 2008 to September 2008

Candidates for blood donation age 4150 years, NRCS, Central Blood Transfusion Service (CBTS), Kathmandu, March 2008 to September 2008

Candidates for blood donation age 5160 years, NRCS, Central Blood Transfusion Service (CBTS), Kathmandu, March 2008 to September 2008

Candidates for blood donation, NRCS, Central Blood Transfusion Service (CBTS), Kathmandu, March 2008 to September 2008

2010 Candidates for blood donation in Banke blood transfusion service, July 2006 to June 2007

Candidates for blood donation in Kaski blood transfusion service, July 2006 to June 2007

Candidates for blood donation in Morang blood transfusion service, July 2006 to June 2007

Candidates for blood donation in Banke, Kaski and Morang blood transfusion services, July 2006 to June 2007

372011 People who inject drugs, age 18-40 years, 2011

2012 Children age 10-12 years born from April 2000 to April 2002, before hepatitis B vaccine introduction, Kathmandu, April 2012

Children age 5-6 years born from April 2006 to April 2007, after hepatitis B vaccine introduction, Kathmandu, April 2012

392012 Patients with ascites attending Nepal Medical College Hospital (NMCTH), Kathmandu, September 2011 to February 2012

2013 People with one or more risk behaviors attending National Public Health Laboratory (NPHL), Kathmandu, November 2011 to May 2012

People living with HIV/AIDS diagnosed among study population, November 2011 to May 2012

412014 People living with HIV/AIDS attending B P Koirala Institute of Health Sciences (BPKIHS), Dharan; Society for Positive Atmosphere and Related Support to HIV and IDS (SPARSHA), Kathmandu; Sukhra Raj Tropical and Infectious Disease Hospital, Teku, Kathmandu, April 2010 to March 2011

People who inject drugs living with HIV/ AIDS attending BPKIHS, Dharan; SPARSHA, Kathmandu; Sukhra Raj Tropical and Infectious Disease Hospital, Teku, Kathmandu, April 2010 to March 2011
205 patients PLWH $24(11.71)^{\mathrm{a}} \quad 137 \mathrm{HBV} / \mathrm{HCV}$ :

(66.83) $10(4.88)$ samples

$(0.17)$

$(0.72)$

2433 blood 3

sample

$19(0.78)$

392 blood

samples

0

$3(0.51)$

$(0.00)$

$(0.51)$

$\begin{array}{llllll}21,716 \text { blood } & 27 & 102(0.46) & - & - & 139 \\ \text { samples } & (0.12) & & & & (0.64)\end{array}$

$-\quad 63(1.21)^{a}$

6

(0.11)

Tiwari BR et

(1)

5995

$21(0.35)^{a}$

10

(0.17)

5351

$-\quad 47(0.88)^{a}$

14

(0.26)

16,557

32

40 people

$-$

1200 children

- $3(0.25)$

2187 children

- $3(0.14)$

43 patients

$-$

678 people

105

(15.49)

7

(17.50)

ANPUD. [86]

$-\quad-$

Upreti SR et al. [35]

105 people PLWH -

14

(13.33)

108 patients PLWH $4(3.70)^{\mathrm{a}}$

$\begin{array}{lll} & & \\ \text { (2.78) } & (0.00) & \text { et al. [15] }\end{array}$ 
Table 1 Studies reporting hepatitis B and C in Nepal (Continued)

\begin{tabular}{|c|c|c|c|c|c|c|c|c|c|c|}
\hline \multirow[t]{2}{*}{$\overline{S N}$} & \multirow[t]{2}{*}{ YEAR } & \multirow[t]{2}{*}{ POPULATION, SITE, TIME } & \multirow{2}{*}{$\begin{array}{l}\text { SAMPLE SIZE, } \\
\mathrm{N}\end{array}$} & \multirow{2}{*}{$\begin{array}{l}\text { ANTI- } \\
\text { HIV(\%) }\end{array}$} & \multicolumn{3}{|l|}{ HBV } & \multirow{2}{*}{$\begin{array}{l}\text { ANTI- } \\
\text { HCV }\end{array}$} & \multirow[t]{2}{*}{ NOTES } & \multirow[t]{2}{*}{ AUTHOR(S) } \\
\hline & & & & & HBSAG(\%) & $\begin{array}{l}\text { ANTI- } \\
\text { HBC(\%) }\end{array}$ & $\begin{array}{l}\text { ANTI- } \\
\text { HBS(\%) }\end{array}$ & & & \\
\hline \multirow[t]{5}{*}{42} & \multirow[t]{5}{*}{2014} & $\begin{array}{l}\text { Women living with HIV/AIDS, } 18 \text { years or } \\
\text { older, February to March } 2010\end{array}$ & 136 women & PLWH & - & - & - & $\begin{array}{l}9 \\
(6.61)\end{array}$ & - & \multirow[t]{5}{*}{$\begin{array}{l}\text { Poudel KC } \\
\text { et al. [39] }\end{array}$} \\
\hline & & $\begin{array}{l}\text { Men living with HIV/AIDS, } 18 \text { years or older, } \\
\text { February to March } 2010\end{array}$ & 183 men & PLWH & - & - & - & $\begin{array}{l}129 \\
(70,49)\end{array}$ & - & \\
\hline & & $\begin{array}{l}\text { People who do not inject drugs living with } \\
\text { HIV/AIDS, } 18 \text { years or older, February to } \\
\text { March } 2010\end{array}$ & 189 people & PLWH & - & - & - & $\begin{array}{l}13 \\
(6.88)\end{array}$ & - & \\
\hline & & $\begin{array}{l}\text { People who inject drugs living with HIV/ } \\
\text { AIDS, } 18 \text { years or older, February to March } \\
2010\end{array}$ & 130 people & PLWH & - & - & - & $\begin{array}{l}125 \\
(95.15)\end{array}$ & - & \\
\hline & & $\begin{array}{l}\text { People living with HIV/AIDS, } 18 \text { years or } \\
\text { older, February to March } 2010\end{array}$ & 319 people & PLWH & - & - & - & $\begin{array}{l}138 \\
(43.26)\end{array}$ & - & \\
\hline \multirow[t]{5}{*}{43} & \multirow[t]{5}{*}{2014} & $\begin{array}{l}\text { Central Blood Transfusion Centre, 2012- } \\
2013\end{array}$ & $\begin{array}{l}67,644 \text { blood } \\
\text { samples }\end{array}$ & $\begin{array}{l}45 \\
(0.07)\end{array}$ & $150(0.22)$ & - & - & $\begin{array}{l}317 \\
(0.47)\end{array}$ & $\begin{array}{l}\text { Syphilis: } 394 \\
\text { (0.58) }\end{array}$ & \multirow[t]{5}{*}{ NRCS [21] } \\
\hline & & $\begin{array}{l}\text { Regional Blood Transfusion Centre, 2012- } \\
2013\end{array}$ & $\begin{array}{l}47,733 \text { blood } \\
\text { samples }\end{array}$ & $\begin{array}{l}40 \\
(0.08)\end{array}$ & $188(0.39)$ & - & - & $\begin{array}{l}126 \\
(0.26)\end{array}$ & $\begin{array}{l}\text { Syphilis: } 284 \\
(0.59)\end{array}$ & \\
\hline & & $\begin{array}{l}\text { District/Emergency Blood Transfusion } \\
\text { Centre, 2012-2013 }\end{array}$ & $\begin{array}{l}61,624 \text { blood } \\
\text { samples }\end{array}$ & $\begin{array}{l}35 \\
(0.06)\end{array}$ & $195(0.32)$ & - & - & $\begin{array}{l}121 \\
(0.20)\end{array}$ & $\begin{array}{l}\text { Syphilis: } 118 \\
(0.19)\end{array}$ & \\
\hline & & Hospital Blood Transfusion Unit, 2012-2013 & $\begin{array}{l}12,320 \text { blood } \\
\text { samples }\end{array}$ & $\begin{array}{l}3 \\
(0.02)\end{array}$ & $32(0.26)$ & - & - & $\begin{array}{l}21 \\
(0.17)\end{array}$ & $\begin{array}{l}\text { Syphilis: } 8 \\
(0.06)\end{array}$ & \\
\hline & & Total, 2012-2013 & $\begin{array}{l}189,321 \text { blood } \\
\text { samples }\end{array}$ & $\begin{array}{l}123 \\
(0.06)\end{array}$ & $565(0.30)$ & - & - & $\begin{array}{l}585 \\
(0.31)\end{array}$ & $\begin{array}{l}\text { Syphilis: } 804 \\
(0.42)\end{array}$ & \\
\hline 44 & 2014 & $\begin{array}{l}\text { Children age 0-15 years with acute } \\
\text { hepatitis attending the liver clinic of Bir } \\
\text { Hospital and Norvic International Hospital of } \\
\text { Kathmandu, Kathmandu, January } 2006 \text { to } \\
\text { December } 2010\end{array}$ & 312 children & - & $15(4.81)^{\mathrm{a}}$ & & & - & - & $\begin{array}{l}\text { Sudhamshu } \\
\text { KC et al. } \\
{[14]}\end{array}$ \\
\hline \multirow[t]{10}{*}{45} & \multirow[t]{10}{*}{2015} & $\begin{array}{l}\text { Women who inject drugs attending } \\
\text { Recovering Nepal services submitted to HIV } \\
\text { testing, Nepalgunj }\end{array}$ & 3 women & $\begin{array}{l}0 \\
(0.00)\end{array}$ & - & - & - & $\begin{array}{l}0 \\
(0.00)\end{array}$ & - & \multirow[t]{10}{*}{$\begin{array}{l}\text { Kinkel HT et } \\
\text { al. [8] }\end{array}$} \\
\hline & & $\begin{array}{l}\text { Men who inject drugs attending Recovering } \\
\text { Nepal services submitted to HIV testing, } \\
\text { Nepalgunj }\end{array}$ & 76 men & $\begin{array}{l}6 \\
(7.89)\end{array}$ & - & - & - & $\begin{array}{l}18 \\
(23.68)\end{array}$ & - & \\
\hline & & $\begin{array}{l}\text { People who inject drugs attending } \\
\text { Recovering Nepal services submitted to HIV } \\
\text { testing, Nepalgunj }\end{array}$ & 79 people & $\begin{array}{l}6 \\
(7.59)\end{array}$ & - & - & - & $\begin{array}{l}18 \\
(22.78)\end{array}$ & - & \\
\hline & & $\begin{array}{l}\text { Women who inject drugs attending } \\
\text { Recovering Nepal services, Dharan; } \\
\text { Biratnagar }\end{array}$ & 69 women & $\begin{array}{l}9 \\
(13.04)\end{array}$ & $3(4.35)$ & - & - & $\begin{array}{l}17 \\
(24.64)\end{array}$ & - & \\
\hline & & $\begin{array}{l}\text { Men who inject drugs attending Recovering } \\
\text { Nepal services, Dharan; Biratnagar }\end{array}$ & 72 men & $\begin{array}{l}12 / 70 \\
(17.14)\end{array}$ & $5(6.94)$ & - & - & $\begin{array}{l}50 \\
(69.44)\end{array}$ & - & \\
\hline & & $\begin{array}{l}\text { People who inject drugs attending } \\
\text { Recovering Nepal services, Dharan; } \\
\text { Biratnagar }\end{array}$ & 141 people & $\begin{array}{l}21 / \\
139 \\
(15.11)\end{array}$ & $8(5.67)$ & - & - & $\begin{array}{l}67 \\
(47.52)\end{array}$ & - & \\
\hline & & $\begin{array}{l}\text { Women who inject drugs attending } \\
\text { Recovering Nepal services submitted to HIV } \\
\text { testing, Kathmandu; Lalitpur; Chitwan }\end{array}$ & 28 women & $\begin{array}{l}0 \\
(0.00)\end{array}$ & $1(3.57)$ & - & - & $\begin{array}{l}2 \\
(7.14)\end{array}$ & - & \\
\hline & & $\begin{array}{l}\text { Men who inject drugs attending Recovering } \\
\text { Nepal services submitted to HIV testing, } \\
\text { Kathmandu; Lalitpur; Chitwan }\end{array}$ & 153 men & $\begin{array}{l}22 / \\
108 \\
(20.37)\end{array}$ & $2(1.31)$ & - & - & $\begin{array}{l}113 \\
(73.86)\end{array}$ & - & \\
\hline & & $\begin{array}{l}\text { People who inject drugs attending } \\
\text { Recovering Nepal services submitted to HIV } \\
\text { testing, Kathmandu; Lalitpur; Chitwan }\end{array}$ & 181 people & $\begin{array}{l}22 / \\
136 \\
(16.18)\end{array}$ & $3(1.66)$ & - & - & $\begin{array}{l}115 \\
(63.53)\end{array}$ & - & \\
\hline & & $\begin{array}{l}\text { Women who inject drugs attending } \\
\text { Recovering Nepal services, Nepalgunj; }\end{array}$ & 100 women & $\begin{array}{l}9 \\
(9.00)\end{array}$ & $4(4.00)$ & - & - & $\begin{array}{l}19 \\
(19.00)\end{array}$ & - & \\
\hline
\end{tabular}


Table 1 Studies reporting hepatitis B and C in Nepal (Continued)

SN YEAR POPULATION, SITE, TIME SAMPLE SIZE,

$\mathrm{N}$

\section{ANTI- \\ HIV(\%)}

\begin{tabular}{llll} 
HBV & & & ANTI- NOTES \\
\cline { 1 - 2 } HBSAG(\%) & $\begin{array}{l}\text { ANTI- } \\
\text { HBC(\%) }\end{array}$ & $\begin{array}{l}\text { ANTI- } \\
\text { HBS(\%) }\end{array}$
\end{tabular}

Dharan; Biratnagar; Kathmandu; Lalitpur;

Chitwan,

Men who inject drugs attending Recovering 301 men

Nepal services submitted to HIV testing,

Nepalgunj; Dharan; Biratnagar; Kathmandu;

Lalitpur; Chitwan,

People who inject drugs attending

Recovering Nepal services submitted to HIV

testing, Nepalgunj; Dharan; Biratnagar;

Kathmandu; Lalitpur; Chitwan,

People who inject drugs attending

Recovering Nepal services, Nepalgunj;

Dharan; Biratnagar; Kathmandu; Lalitpur;

Chitwan,

462015 Patients attending Manipal Teaching Hospital, Pokhara, 2008 to 2013

People living with HIV/AIDS at Manipal Teaching Hospital, Pokhara, 2008 to 2013

47

\footnotetext{
2015 Boys and men 15+ years who inject drugs, Sunsari, Morang and Jhapa districts, July 2015
}

354 people

401 people

$92 /$
397
$(23.17)$

25,708

individual

218

blood

samples

blood

samples

360 boys and 30

30
$(8.33)$

$3(0.83)$

men

(8.33)

$40 / \quad 10(3.32)$
254
$(15.75)$
$49 \quad-$
$(13.84)$

181

(60.13)

2015 Boys and men 16+ years who inject drugs, Kathmandu, Lalitpur and Bhaktapur districts, men

340 boys and 22

22
$(6.47)$

$0(0.00)$ Kathmandu Valley, June 2015 to July 2015

49

2015 Boys and men 16+ years who inject drugs, Pokhara Valley, June 2015 to July 2015

$$
\begin{array}{lll}
\begin{array}{l}
345 \text { boys and } \\
\text { men }
\end{array} & \begin{array}{l}
10 \\
(2.90)
\end{array} & 6(1.74) \\
140 \text { people } & \text { PLWH } & 5(3.57) \\
137 \text { people } & \text { PLWH } & 7(5.11) \\
203 \text { people } & \text { PLWH } & 13(6.40) \\
51 \text { people } & \text { PLWH } & 0(0.00) \\
146 \text { people } & \text { PLWH } & 5(3.42) \\
677 \text { people } & \text { PLWH } & 30(4.43)
\end{array}
$$

345 boys and$$
10 \quad 6(1.74)
$$$$
\text { (2.90) }
$$$$
\text { PLWH } 5 \text { (3.57) }
$$$$
\text { PLWH } 7 \text { (5.11) }
$$$$
\text { PLWH } 13 \text { (6.40) }
$$$$
146 \text { people }
$$$$
\text { PLWH } 5 \text { (3.42) }
$$$$
562 \text { people PLWH } 8(1.42)
$$$$
\text { AIDS in all Development Regions, }
$$$$
\text { Sex workers living with HIV/AIDS in all }
$$$$
\text { Development Regions, }
$$

Migrant workers living with HIV/AIDS in all Development Regions,

Gay, Lesbian and Transgender people living with HIV/AIDS in all Development Regions,

Non most at risk population living with HIV/ AIDS in all Development Regions, 
Table 1 Studies reporting hepatitis B and C in Nepal (Continued)

\begin{tabular}{|c|c|c|c|c|c|c|c|c|c|c|}
\hline \multirow[t]{2}{*}{ SN } & \multirow[t]{2}{*}{ YEAR } & \multirow[t]{2}{*}{ POPULATION, SITE, TIME } & \multirow{2}{*}{$\begin{array}{l}\text { SAMPLE SIZE, } \\
\mathrm{N}\end{array}$} & \multirow{2}{*}{$\begin{array}{l}\text { ANTI- } \\
\text { HIV(\%) }\end{array}$} & \multicolumn{3}{|l|}{ HBV } & \multirow{2}{*}{$\begin{array}{l}\text { ANTI- } \\
\text { HCV }\end{array}$} & \multirow[t]{2}{*}{ NOTES } & \multirow[t]{2}{*}{ AUTHOR(S) } \\
\hline & & & & & HBSAG(\%) & $\begin{array}{l}\text { ANTI- } \\
\text { HBC(\%) }\end{array}$ & $\begin{array}{l}\text { ANTI- } \\
\text { HBS(\%) }\end{array}$ & & & \\
\hline$\overline{51}$ & 2016 & $\begin{array}{l}\text { People who inject drugs with last 30-day } \\
\text { frequent injection drug use attending re- } \\
\text { habilitation centers, Kathmandu; Bhaktapur; } \\
\text { Lalitupur; Sindupalchowk }\end{array}$ & 167 people & - & - & - & - & $\begin{array}{l}20 / 87 \\
(22.99)\end{array}$ & - & $\begin{array}{l}\text { Loewinger } \\
\text { G et al. [94] }\end{array}$ \\
\hline 52 & 2016 & $\begin{array}{l}\text { Girls and women 16+ years who inject } \\
\text { drugs, Kathmandu, Lalitpur and Bhaktapur } \\
\text { districts, Kathmandu Valley, April } 2016 \text { to } \\
\text { July } 2016\end{array}$ & $\begin{array}{l}160 \text { girls and } \\
\text { women }\end{array}$ & $\begin{array}{l}14 \\
(8.75)\end{array}$ & $3(1.87)$ & - & - & $\begin{array}{l}34 \\
(21.25)\end{array}$ & $12(7.50)$ & $\begin{array}{l}\mathrm{MoH} / \\
\text { NCASC [95] }\end{array}$ \\
\hline 53 & 2016 & $\begin{array}{l}\text { Boys and men } 15+\text { years who inject drugs, } \\
\text { Rupandehi, Kapilvastu, Dang, Banke, Kailali } \\
\text { and Kanchanpur districts }\end{array}$ & $\begin{array}{l}300 \text { boys and } \\
\text { men }\end{array}$ & $\begin{array}{l}7 \\
(2.33)\end{array}$ & $5(1.67)$ & - & - & $\begin{array}{l}24 \\
(8.00)\end{array}$ & $\begin{array}{l}\text { Syphilis: } 1 \\
(0.33) \text {; } \\
\text { Syphilis } \\
\text { History: } 5 \\
(1.67)\end{array}$ & $\begin{array}{l}\mathrm{MoH} / \\
\text { NCASC [96] }\end{array}$ \\
\hline 54 & 2016 & $\begin{array}{l}\text { Patients who came in contact with HIV or } \\
\text { other chronic liver disease and jaundice } \\
\text { attending Teku Hospital, Tribhuvan } \\
\text { University Teaching Hospital, NRCS }\end{array}$ & 2700 patients & - & - & - & - & $\begin{array}{l}100 \\
(3.70)\end{array}$ & - & $\begin{array}{l}\text { Nepal A et } \\
\text { al. [97] }\end{array}$ \\
\hline \multirow[t]{5}{*}{55} & 2016 & $\begin{array}{l}\text { Central Blood Transfusion Centre, 2014- } \\
2015\end{array}$ & $\begin{array}{l}69,303 \text { blood } \\
\text { samples }\end{array}$ & $\begin{array}{l}21 \\
(0.03)\end{array}$ & $192(0.28)$ & - & - & $\begin{array}{l}224 \\
(0.32)\end{array}$ & $\begin{array}{l}\text { Syphilis: } 360 \\
(0.52)\end{array}$ & \multirow[t]{5}{*}{ NRCS [22] } \\
\hline & & $\begin{array}{l}\text { Regional Blood Transfusion Centre, 2014- } \\
2015\end{array}$ & $\begin{array}{l}42,511 \text { blood } \\
\text { samples }\end{array}$ & $\begin{array}{l}13 \\
(0.03)\end{array}$ & $151(0.35)$ & - & - & $\begin{array}{l}56 \\
(0.23)\end{array}$ & $\begin{array}{l}\text { Syphilis: } 115 \\
(0.27)\end{array}$ & \\
\hline & & $\begin{array}{l}\text { District/Emergency Blood Transfusion } \\
\text { Centre, 2014-2015 }\end{array}$ & $\begin{array}{l}77,016 \text { blood } \\
\text { samples }\end{array}$ & $\begin{array}{l}27 \\
(0.03)\end{array}$ & $227(0.29)$ & - & - & $\begin{array}{l}119 \\
(0.15)\end{array}$ & $\begin{array}{l}\text { Syphilis: } 260 \\
(0.34)\end{array}$ & \\
\hline & & Hospital Blood Transfusion unit, 2014-2015 & $\begin{array}{l}28,324 \text { blood } \\
\text { samples }\end{array}$ & $\begin{array}{l}7 \\
(0.02)\end{array}$ & $47(0.16)$ & - & - & $\begin{array}{l}23 \\
(0.08)\end{array}$ & $\begin{array}{l}\text { Syphilis: } 25 \\
(0.09)\end{array}$ & \\
\hline & & Total, 2014-2015 & $\begin{array}{l}217,154 \text { blood } \\
\text { samples }\end{array}$ & $\begin{array}{l}68 \\
(0.03)\end{array}$ & $617(0.28)$ & - & - & $\begin{array}{l}422 \\
(0.19)\end{array}$ & $\begin{array}{l}\text { Syphilis: } 760 \\
(0.35)\end{array}$ & \\
\hline
\end{tabular}

${ }^{a}$ : Study describes seroprevalence of active HBV infection. Test(s) used in the survey is(are) not specified

b. Study describes seroprevalence of exposure to HBV. Test(s) used in the survey is (are) not specified

0.33, respectively), [20] and Kinkel HT et a, between male and female PWID attending Recovering Nepal services in 2015 (19.00 vs. 60.13).

Age has been fundamental to the design of hepatitis $C$ public health policies in many countries. It is known that age can relate to many factors in the epidemic: year of introduction of the virus, availability of tests and distribution of contaminated blood products, cumulative exposure (such as injection drug use, unprotected sexual activity across adult life), and status of harm reduction

Table 2 Findings of the meta-analyses for hepatitis $\mathrm{C}$ virus (HCV) prevalence measures

\begin{tabular}{|c|c|c|c|c|c|c|c|c|}
\hline \multirow[t]{2}{*}{ Populations at risk } & \multirow{2}{*}{$\begin{array}{l}\text { Studies } \\
\text { Total } \\
\mathrm{N}\end{array}$} & \multirow{2}{*}{$\begin{array}{l}\text { Samples } \\
\text { Total N }\end{array}$} & \multicolumn{2}{|c|}{$\begin{array}{l}\text { HCV prevalence } \\
\text { estimates }\end{array}$} & \multicolumn{4}{|c|}{ Heterogeneity measures } \\
\hline & & & $\begin{array}{l}\text { Mean } \\
(\%)\end{array}$ & $95 \% \mathrm{Cl}$ & $\mathrm{Q}(p \text {-value })^{\mathrm{a}}$ & $\tau^{2 b}$ & $\begin{array}{l}I^{2}(\text { confidence limits- } \\
\%)^{c}\end{array}$ & $\begin{array}{l}\text { Prediction interval } \\
(\%)^{d}\end{array}$ \\
\hline People who inject drugs & 15 & 3140 & 45.17 & $\begin{array}{l}26.34- \\
64.73\end{array}$ & $\begin{array}{l}1714.1(< \\
0.0001)\end{array}$ & 0.1487 & $99.2(99.0-99.3)$ & $0-100$ \\
\hline Populations at intermediate risk & 12 & 4998 & 12.76 & $\begin{array}{l}5.44- \\
22.47\end{array}$ & $\begin{array}{l}668.83(< \\
0.0001)\end{array}$ & 0.0486 & $98.4(97.9-98.7)$ & $0-59.58$ \\
\hline $\begin{array}{l}\text { Populations at low risk (general } \\
\text { population) }\end{array}$ & 28 & 972,123 & 0.68 & $0.54-0.86$ & $\begin{array}{l}683.44(< \\
0.0001)\end{array}$ & 0.2027 & $96.0(95.1-96.8)$ & $0.26-1.75$ \\
\hline $\begin{array}{l}\text { Populations with liver-related } \\
\text { conditions }\end{array}$ & 6 & 411 & 11.51 & $\begin{array}{l}7.73- \\
15.87\end{array}$ & $7.40(0.1926)$ & 0.0018 & $32.4(0-72.7)$ & $3.48-22.89$ \\
\hline Special clinical populations & 3 & 133 & 1.67 & $0-5.81$ & $2.79(0.2473)$ & 0.0022 & $28.4(0-92.6)$ & $0-75.38$ \\
\hline
\end{tabular}

${ }^{a} \mathrm{Q}$ : the Cochran's Q statistic is a measure assessing the existence of heterogeneity in HCV prevalence estimates

${ }^{\mathrm{b}} \tau^{2}$ : the estimated between-study variance in the double arcsine transformed proportions of the true HCV prevalence estimates. The back-transformed $\tau^{2}$ was not calculated as the methodology to do so is not currently available

$\mathrm{q}^{2}$ : a measure assessing the magnitude of between-study variation that is due to differences in HCV prevalence estimates across studies rather than chance

${ }^{d}$ Prediction interval: estimates the $95 \%$ interval in which the true HCV prevalence in a new HCV study will lie 
strategies [41-43]. In Nepal, age and hepatitis C have only been featured in two studies. De Bruyn et al. found anti-HCV to be positive in $11.8 \%$ of patients age $16-$ 65 years attending Amp Pipal Hospital at Gorkha district in 1993; and Sawayama Y et al. found $2.27 \%$ in female villagers 45-54 years from Bhadrakali and Kotyang villages in 1996 [40, 44].

\section{HIV co-infection prevalence}

The first HIV prevalence study in Nepal dates 1989, [44] 1 year after the first case of HIV was detected in the country [45]. So far, eighteen prevalence studies also assessed at some time the HIV infection in their population, almost half a million people and roughly half of the population evaluated for HBV or HCV infection since 1985, the year of debut of anti-HIV ELISA tests.

As stated previously for overall population, candidates for blood donation represent the majority of the population tested for HIV co-infection in Nepal in the fifty-five viral hepatitis prevalence studies of this review. Yet, regardless of age and/or gender subgroups, candidates for blood donation have failed to present HIV seropositivity rates above $0.2 \%[20,12,18]$, constituting themselves a population of low prevalence for HIV co-infection. This data must not considered a proxy for general population, as candidates for blood donation have shown to be a "poor control group for non-genetic studies of diseases related to environmentally, behaviourally, or socially patterned exposures", [46] but a reason to pursue further detection of viral hepatitis in priority populations.

As we evaluate the remaining studied population, we find that the highest rates of HIV infection found in this review do not belong to PWID, but sex trafficked girls and women, at an approximate 30\% rate of infection in 2008 (74/246) [7). This is closely followed by PWID attending Recovering Nepal services, if accounted the participation of those with previously defined HIV status, with rates as high as $23.17 \%$ in 2015. Such findings are consonant with latest numbers of Family Health International data for HIV prevalence in PWID in Kathmandu (21\% in 2009) [3] and increase of HIV and sexually transmitted infections in survivors of sex traffic, particularly for Nepalese girls and women [47-49].

\section{Discussion}

This review collects all available surveys performed in Nepal or with Nepalese population. It provides relevant information to policy makers, researchers and activists.

\section{Developments of improved strategic information}

It has been 44 years since the first viral hepatitis prevalence study took place in Nepal. Since then, sixty different publications, of which fifty-five are available in this review, have dedicated themselves to the better understanding of these epidemics.
Almost a quarter of these scientific publications and reports have been issued in the last 3 years. While there is still much to investigate, it is undeniable that Nepal's civil society organizations and academia have been successful in their struggle to improve the reactive approach to viral hepatitis and HIV, shaping public health policy and visibility of key populations.

Superior strategic information and overall engagement to the epidemics lead to additional victories: the inclusion of LGBT issues in government policies, the return of harm reductions strategy in 2007, increase in donor funding for the response to HIV, and stronger ties between emerging and existing networks of key populations and representatives, healthcare professionals, academia, UNAIDS and GoN [50-55]. Such echoing common voice for change lead to the preliminary discussion of National Viral Hepatitis Guidelines and negotiation of licensed generic drugs for hepatitis $C$ treatment at a fraction of prices offered to other developing countries [41, 56-59]. This recent collective represents a cornerstone for viral hepatitis in Nepal.

\section{Gaps and key populations}

It would seem to be that the shared modes of transmission of viral hepatitis and HIV, and the resulting similar epidemiological profiles, could translate into one equally successful public health intervention for both epidemics.

This is hardly the truth. Regardless of improvements in blood safety, availability of harm reduction services and assistance to sexually transmitted and reproductive tract infections, with significant drops in HIV prevalence since the last decade, many key populations sustain subpar decrease in hepatitis $C$ numbers. Such is the case of PWID in Nepal: hepatitis $\mathrm{C}$ exceeds three times the HIV prevalence in several cohorts.

Since 2000, PWID, PLHIV, sex workers and LGBT have figured in no less than seventeen different viral hepatitis prevalence studies, almost a third of studies collected in this review, contributing without precedents to the national strategic information and deeper understanding of the response to public health interventions. Additional publications also developed initial data for other population groups such as refugees/misplaced persons and survivors of sex traffic.

Our research could not retrieve any studies regarding transgender population, male sex workers or incarcerated persons in Nepal.

\section{Hepatitis B vaccine coverage and elimination of vertical transmission (EVT)}

Hepatitis B is a highly contagious, yet preventable disease $[60,61]$. In recent years, many countries have chosen to scale up maternal and newborn care in order to secure a generation 'free of hepatitis B'. 
Our review provided two studies depicting hepatitis B in infancy in Nepal. The first study states hepatitis B is responsible for approximately $5 \%$ of the cases of acute hepatitis in children 0-15 years [14]. The second study describes observed benefits of hepatitis $B$ vaccine introduction to children in Nepal in 2002.

While no routine for hepatitis B immunoglobulin in prevention of vertical transmission has been implemented in Nepal, the rates of acute hepatitis related to HBV infection in children are quite different than the prevalence values of HBV in pregnant women $(0.32 \%)$, an indicator that perhaps household exposure during infancy, and not mother-tochild transmission, is the reason for new but likely overlooked exposure during infancy (households).

Commercial vaccines for hepatitis B have been available worldwide since 1981 [62]. In Nepal, however, it has only been introduced in 2002. After fifteen years and despite recommendations issued by GoN, the country still struggles to provide appropriate vaccine coverage [35, 63].

Official government reports state that from 2002 until 2009, third-dose hepatitis B vaccine coverage for children 12-23 months stood at approximately 80\%. [63, 64] World Health Organization (WHO) and United Nations Children's Fund (UNICEF) estimate improved coverage in 20,012 and 2013, slightly above 95\%. [65] Such higher vaccine coverage numbers are not homogenous in Nepal - not all municipalities have immunization plans or appropriate structure at their disposal $[63,64]$. Moreover, the intervention requires timely birth-dose vaccination for a most successful response, [66] and faces many other obstacles [67].

Hepatitis B vaccine is available to healthcare workers $(\mathrm{HCW})$ in many countries. In Nepal, HCW are featured in two studies as an alternative to controls, with HBsAg seropositivity rates of 1.38 and $1.69 \%$ [26, 68]. These figures are lower than the ones presented by key populations, but still higher than those of candidates for blood donation. Further investigation reveals that $\mathrm{HCW}$ and students in Nepal have largely mishandled biosafety procedures while at high risk of exposure to the infective agent. Studies in tertiary care centers have shown frequent needle-stick and sharps-related injuries, and incomplete or fully ignored vaccination and post prophylaxis procedures [69-71].

Nevertheless, UNAIDS understands that the strengthening of the immunization plan and maternal health must be accompanied of a nationwide awareness campaign for $\mathrm{HCW}$ and future health professionals.

\section{Community and the strengthening of health systems}

Communities were the first responders to the HIV epidemic, nearly thirty years ago. They have continuously played an essential role in development of research, health services and shaping of public health policy worldwide, expanding their activities to sustainable and affordable vaccines and medicines for viral hepatitis.
Moreover, these collectives of advocates, researchers, clients or providers have the ability to work unisonous with marginalized populations, increasing the reach and quality of health systems and health services, often detecting overlooked issues such as stigma and discrimination. Whether leading research or promoting health services, civil society engagement improves awareness, prevention, diagnosis and retention in care. This has been the case of both viral hepatitis and HIV epidemic.

In Nepal, these initiatives are considered to be just as important as developments provided by academia, government and international agencies. It is clear that these endeavors provide a unique opportunity to fill critical gaps such as strategic information, low immunization coverage rates and elimination of mother-to-child and household transmission.

\section{Conclusion}

The present review illustrates different turning points in viral hepatitis and HIV co-infection epidemiology in Nepal. Since 1973, when the first study on viral hepatitis in the country was published, there have been many changes in the understanding of these epidemics.

These include the indirect effects of successful public health policies aimed towards HIV, such as the decrease of viral hepatitis prevalence in PWID, and their limitations, revealing overlooked population groups and issues in viral hepatitis that require public health policies of their own.

The review also allows one to witness the progressive scientific development by Nepalese researchers and institutions, and civil society representatives' participation. Such collaboration correlates with increased number of studies and sample sizes in recent years including the survey of key populations, and will be fundamental for the success of the National HIV Strategic Plan 2016-2021 and achieving the SDG by 2030.

\section{Acknowledgements \\ We are grateful for the support provided by the Biostatistics, Epidemiology, and Biomathematics Research Core at the Weill Cornell Medical College in Qatar. The authors are also grateful for the valuable suggestions and comments by the reviewers of this article.}

Availability of data and materials

All data present in this review is accessible through scientific journals and reports.

Authors' contributions

MCMN and KB performed the systematic review. KB supervised the research and provided inputs for discussion. All authors contributed equally to the discussion, conclusion and review of this manuscript. All authors read and approved the final manuscript.

Ethics approval and consent to participate

This study does not require ethics committee approval or written informed consent as it relies entirely on published available data.

Consent for publication

Not applicable. 


\section{Competing interests}

The authors declare that they have no competing interests.

\section{Publisher's Note}

Springer Nature remains neutral with regard to jurisdictional claims in published maps and institutional affiliations.

\section{Received: 13 December 2017 Accepted: 28 August 2018}

\section{Published online: 06 September 2018}

\section{References}

1. Kleinman S, Busch M. The risk of transfusion-transmitted infection: direct estimation and mathematical modelling. Ballière's Clinical Haematology. 2000;13(4):631-49.

2. Government of Nepal. Ministry of Health and Population. National Centre for AIDS and STD Control. Country Progress Report Nepal. 2015.

3. Narain JP. Three decades of HIV/AIDS in Asia. New Delhi: Vivek Mehra for SAGE Publications India Pvt Ltd; 2012

4. Government of Nepal. National Planning Comission. Sustainable development goals 2016-2030. Kathmandu: National Preliminary Report; 2015.

5. Government of Nepal. Ministry of Health. National Centre for AIDS and STD Control. Kathmandu: Nepal HIVision 2020 - Ending the AIDS epidemic as a public health threat, by 2030; 2016

6. Hillis A, Shrestha S, Saha N. An epidemic of infectious hepatitis in the Kathmandu Valley. J Nepal Med Assoc. 1973;11(5):145-53.

7. Silverman JG, Decker MR, Gupta J, Dharmadhikari A, Seage G, Raj A. Syphilis and hepatitis co-infection among HIV-infected, sex-trafficked women and girls, Nepal. Emerg Infect Dis. 2008;14(6):932-4.

8. Kinkel HT, Karmacharya D, Shakya J, Manandhar S, Panthi S, Karmacharya P et al. Prevalence of HIV, hepatitis B and C infections and an assessment of HCV-genotypes and two IL28B SNPs among people who inject drugs in three regions of Nepal. PLOS One. 2015;10(8):1-18.

9. Moher D, Liberati A, Tetzlaff J, Altman DG, PRISMA Group. Preferred reporting items for systematic reviews and meta-analyses: the PRISMA statement. PLoS Med. 2009:6(7):e1000097.

10. Nuti M. The low prevalence of HBV markers in Nepal. Transact R Soc Trop Med Hyg. 1988:82:144.

11. Dhungel B, Dhungel K, Easow J, Singh Y. Opportunistic infection among HIV seropositive cases in Manipal Teaching Hospital, Pokhara, Nepal. Kathmandu Univ Med J. 2008;6(3):335-9.

12. Karki S, Ghimire P, Tiwari BR, Shrestha AC, Gautam A, Rajkarnikar M. Seroprevalence of HIV and hepatitis C co-infection among blood donors in Kathmandu Valley, Nepal. Southeast Asian J Trop Med Public Health. 2009:40(1):67-70

13. Tiwari B, Ghimire P, Kandel S, Rajkarnikar M. Seroprevalence of HBV and HCV in blood donors: a study from regional blood transfusion services of Nepal. Asian J Transfus Sci. 2010;4(2):91-3.

14. Sudhamshu K, Sharma D, Poudyal N, Basnet BK. Acute viral hepatitis in pediatric age groups. J Nepal Med Assoc. 2014;52(193):687-91.

15. Barnawal SP, Niraula SR, Agrahari AK, Bista N, Jha N, Pokharel PK. Human immunodeficiency virus and hepatitis C virus coinfection in Nepal. Indian J Gastroenterol. 2014;33(2):141-5.

16. Shrestha SM, Shrestha S, Tsuda F, Sawada N, Tanaka T, Okamoto H, et al. Infection with $G B$ virus $C$ and hepatitis $C$ virus in drug addicts, patients on maintenance hemodialysis, or with chronic liver disease in Nepal. J Med Virol. 1997;53:157-61

17. Mohamoud YA, Mumtaz GR, Riome S, Miller D, Abu-Raddad LI. The epidemiology of hepatitis C virus in Egypt: a systematic review and data synthesis. BMC Infectious Diseases. 2013;13:288. https://doi.org/10.1186/ 1471-2334-13-288.

18. Chander A, Pahwa V. Status of infectious disease markers among blood donors in a teaching hospital, Bhairahawa, western Nepal. J Commun Dis. 2003;35(3):188-97.

19. Karki S, Ghimire P, Tiwari BR, Maharjan A, Rajkarnikar M. Trends in hepatitis B and hepatitis $C$ seroprevalence among Nepalese blood donors. Jpn J Infect Dis. 2008:61:324-6.

20. Shrestha AC, Ghimre P, Tiwari BR, Rajkarnikar M. Transfusion-transmissible infections among blood donors in Kathmandu, Nepal. J Infect Dev Ctries. 2009;3(10):794-7.

21. Nepal Red Cross Society National Blood Transfusion Service. Annual Progress Report 2069/070 (2012/13)
22. Nepal Red Cross Society. Annual Progress Report 2071/072 (2014/15).

23. Chatterjee A, Uprety L, Chapagain M, Kafle K. Drug abuse in Nepal: a rapid assessment study. Bull Narc. 1996;48(1):11-33.

24. Lam L. Comments on Strang et al.'s "Heroin smoking by 'chasing the dragon': origins and history". Addiction. 1997;92(6):685-95. https:// onlinelibrary.wiley.com/doi/pdf/10.1080/09652149737665.

25. Shrestha S, Shrestha D, Gafney T, Maharjan K, Tsuda F, Okamoto H. Hepatitis B and C infection among drug abusers in Nepal. Trop Gastroenterol. 1996;17(4):212-3.

26. Shrestha SM, Tsuda F, Okamoto H, Tokita H, Horikita M, Tanaka T, et al. Hepatitis B virus subtypes and hepatitis C virus genotypes in patients with chronic liver disease in Nepal. Hepatol. 1994;19(4):805-9.

27. Shrestha SM, Shrestha S, Shreshta A, Tsuda F, Endo K, Takahashi M, et al. High prevalence of hepatitis $B$ virus infection and inferior vena cava obstruction among patients with liver cirrhosis or hepatocellular carcinoma in Nepal. Hepatol. 2007;22:1921-8.

28. Kane MA, Bradley DW, Shrestha SM, Maynard J, Cook EH, Mishra RP, et al. Recovery of a possible etiologic agent and transmission studies in marmosets. JAMA. 1984:252(22):3140-5.

29. Shrestha SM, Shrestha I, Maharjan K. Family clustering of HBV infection in the household of persistent HBsAg carriers: spread of HBV by horizontal transmission. J Inst Med. 1991;13:319-26.

30. Shrestha SM. Seroepidemiology of viral hepatitis in Surkhet, Nepal. J Inst Med. 1987;9:1-10.

31. Poudel KC, Jimba M, Okumura J, Wakai S. Emerging co-infection of HIV and hepatitis B virus in far western Nepal. Trop Doct. 2006;36:186-7.

32. Goh K, Kong K, Heng B, Oon C. Seroepidemiology of hepatitis A and hepatitis B virus infection in a Gurkha Community in Singapore. J Med Virol. 1993:41:146-9

33. Shrestha SM, Shrestha S. Chronic hepatitis B in Nepal: an Asian country with low prevalence of HBV infection. Trop Gastroenterol. 2012;33(2):95-101.

34. Joshi S, Ghimire G. Serological prevalence of antibodies to Human Immunodeficiency Virus (HIV) and hepatitis B virus (HBV) among healthy Nepalese males - a retrospective study. Kathmandu Univ Med J. 2003;1(4):251-5.

35. Upreti SR, Gurung S, Patel M, Dixit S, Krause K, Shakya G, et al. Prevalence of chronic hepatitis $B$ virus infection before and after implementation of a hepatitis B vaccination program among children in Nepal. Vaccine. 2014; 32(34):4304-9.

36. Lavanchy D. The global burden of hepatitis C. Liver Int. 2009;29(Suppl 1):74-81.

37. Sulkowski M, Thomas DL. Hepatitis in the HIV-infected person. Ann Intern Med. 2003;138(3):197-208

38. Shrestha IL. Seroprevalence of antibodies to hepatitis $C$ virus among injecting drug users from Kathmandu. Kathmandu Univ Med J. 2003;1(2):101-3.

39. Poudel KC, Palmer PH, Jimba M, Mizoue T, Kobayashi J, Poudel-Tandukar K. Coinfection with hepatitis C virus among HIV-positive people in Kathmandu Valley, Nepal. J Int Assoc Provid AIDS Care. 2014;13(3):277-83.

40. Sawayama Y, Hayashi J, Ariyama I, Furusyo N, Kawasaki T, Kawasaki M, et al. A ten year serological survey of hepatitis $A, B$ and $C$ viruses infections in Nepal. J Epidemiol. 1999;9(5):350-4.

41. Mesquita F, Santos MÉ, Benzaken A, Corrêa R, Cattapan E, Sereno L, et al. The Brazilian comprehensive response to hepatitis $C$ : from strategic thinking to access to interferon-free therapy. BMC Public Health. 2016;16(1132)

42. European Centre fr Disease Prevention and Control. Hepatitis C surveillance in Europe 2013. Stockholm: ECDC; 2015.

43. U.S. Department of Health and Human Services. Centers for Disease Control and Prevention. Hepatitis C - why people born from 1945-1965 should get tested. United States: CDC; 2016

44. de Bruyn G, Song E. Seroepidemiology of hepatotropic viral infections in Amp Pipal, Nepal. Trop Doct. 1998;28:173-4.

45. Hannun J. AIDS in Nepal: communities confronting an emerging epidemic. New York: AmFAR in Association with Seven Stories Press; 1997.

46. Golding J, Northstone K, Miller L, Davey Smith G, Pembrey M. Differences between blood donors and a population sample: implications for casecontrol studies. Int J Epidemiol. 2013:42(4):1145-56.

47. Joffres $C$, Mills E, Joffres M, Khanna T, Walia H, Grund D. Sexual slavery without borders: trafficking for commercial sexual exploitation in India. Int J Equity Health. 2008;7(22)

48. Sarkar K, Bal B, Mukherjee R, Chakraborty S, Saha S, Ghosh A, et al. Sextrafficking, violence, negotiating skill, and HIV infection in brother-based sex workers of Eastern India, adjoining Nepal, Bhutan, and Bangladesh. J Health Popul Nutr. 2008;26(2):223-31. 
49. Silverman J, Decker M, Gupta J, Maheshwari A, Willis B, Raj A. HIV prevalence and predictors of infection in sex-trafficked Nepalese girls and women. JAMA. 2007;298(5):536-42.

50. UNDP, USAID. Being LGBT in Asia. Bangkok: Nepal Country Report; 2014.

51. Federal Ministry for Economic Cooperation and Development. Division for Health and Population. Opioid substitution therapy in Nepal. Bonn: Deutsche Gesellschaft für Internationale Zusammenarbeit (GIZ) GmbH; 2016.

52. UNDP, Williams Institute. Surveying Nepal's sexual and gender minorities: an inclusive approach. Bangkok: UNDP; 2014

53. UNDP. Lost in transition: transgender people, rights and HIV vulnerability in the Asia-Pacific Region. Bangkok: UNDP; 2012.

54. Ministry of Health and Population. National Centre for AIDS and STI Control. National targeted intervention operational guidelines - injecting drug users. 2010

55. Thomson N. Harm reduction history, response, and current trends in Asia. J Food Drug Anal. 2013;21(Suppl 4):113-6.

56. Natco Pharma. News \& Announcements. 2017. http://natcopharma.co.in/ about/news/. Accessed 21 Feb 2017.

57. Gilead Sciences, Inc. Chronic hepatitis C treatment expansion - generic manufacturing for developing countries. 2015.

58. Bristol-Myers Squibb Company, Medicines Patent Pool. Medicines Patent Pool. 2015. https://medicinespatentpool.org/uploads/2015/11/MPP-HCVLicense-Agreement-BMS-FINAL-Web-00000002.pdf. Accessed 21 Feb 2017.

59. Iyengar S, Tay-Teo K, Vogler S, Beyer P, Wiktor S, de Joncheere K, et al. Prices, costs, and affordability of new medicines for hepatitis $C$ in 30 countries: an economic analysis. PLOS Med. 2016;13(5):e1002032.

60. Lavanchy D. Chronic viral hepatitis as a public health issue in the world. Best Pract Res Clin Gastroenterol. 2008;22(6):991-1008.

61. Mandell G, Bennett J, Mandell DR. In: Mandell JEBRD GL, editor. Douglas, and Bennett's Principles and Practice of Infectious Diseases. 7th ed. Philadelphia: Elsevier; 2010.

62. Blumberg BS. The discovery of the hepatitis B virus and the invention of the vaccine: a scientific memoir. J Gastroenterol Hepatol. 2002;17(Suppl):502-3.

63. Ministry of Health \& Population. Department of Health Services. Child Health Division. National immunization program - reaching every child comprehensive multi-year plan 2068/2072 (2011-2016). Kathmandu: Government of Nepal; 2011.

64. Ministry of Health. Department of Health Services. Child Health Division. National immunization program of Nepal - reaching every village - multiyear plan of action 2007-2011. Kathmandu: Government of Nepal; 2007.

65. Berger S. Hepatitis B: global status: GIDEON Informatics Inc; 2016.

66. World Health Organization. Global health sector strategy on viral hepatitis, 2016-2021. Geneva: World Health Organization; 2016.

67. Tajiri K, Shimizu Y. Unsolved problems and future perspectives of hepatitis B virus vaccination. World J Gastroenterol. 2015;21(23):7074-83.

68. Shrestha S, Bhattarai M. Study of hepatitis B among different categories of health care workers. J Coll Physicians Surg Pak. 2006;16(2):108-11.

69. Bhattarai S, Smriti K, Pradhan PM, Lama S, Rijal S. Hepatitis B vaccination status and needle-stick and sharps-related injuries among medical school students in Nepal: a cross-sectional study. BMC Res Notes. 2014;7(774)

70. Gurubacharya D, Mathura K, Karki D. Knowledge, attitude and practices among health care workers on needle-stick injuries. Kathmandu Univ Med J. 2003;1(2):91-4

71. Singh B, Paudel B, KC S. Knowledge and practice of health care workers regarding needle stick injuries in a tertiary care center of Nepal. Kathmandu Univ Med J. 2015;13:230-3.

72. Shrestha SM. Acute sporadic viral hepatitis in Nepal. Trop Gastroenterol. 1987:8:99-105

73. Reynolds RD, Moser P, Acharya S, McConnell W, Andon M, Howard MP. Nutritional and medical status of lactating women and their infants in the Kathmandu valley of Nepal. Am J Clin Nutr. 1988;47:722-8.

74. Mertens T, Tondorf G, Siebolds M, Kruppenbacher J, Shrestha S, Mauff G, et al. Epidemiology of HIV and hepatitis B virus (HBV) in selected African and Asian populations. Infection. 1989;17(1):4-7.

75. Shrestha SM. Seroepidemiology of hepatitis B in Nepal. J Commun Dis. 1990;22:27-32.

76. Bhatta P, Thapa S, Neupane S, Baker J, Friedman M. Commercial sex workers in Kathmandu Valley: profile and prevalence of sexually transmitted diseases. J Nepal Med Assoc. 1994;32(111):191-203.

77. Rai SK, Shibata H, Satoh M, Murakoso K, Sumi K, Kubo T, et al. Seroprevalence of hepatitis B and C viruses in Eastern Nepal. Kansenshogaku Zasshi. 1994;68(12):1492-7.
78. Nakashima K, Kashiwagi S, Noguchi A, Hirata M, Hayashi J, Kawasaki T, et al. Human T-lymphotropic virus type-I, and hepatitis A, B and C viruses in Nepal: a serological survey. J Trop Med Hyg. 1995;98(5):347-50.

79. Manandhar K, Shrestha B. Prevalence of HBV infection among the healthy Nepalese males: a serological survey. J Epidemiol. 2000;10(6):410-3.

80. Manandhar K, Shrestha B. Prevalence of hepatitis B virus infection amongst healthy Nepalese males. Trop Gastroenterol. 1998;19(4):145-7.

81. Bidya S. HBsAg carriers among healthy Nepalese men: a serological survey. J Health Popul Nutr. 2002;20(3):235-8. http://www.recoveringnepal.org.np/ image/info_materials/20111103081156838553767.pdf.

82. Chiba H, Takezaki T, Neupani D, Kim J, Yoshida S, Mizoguchi E, et al. An epidemiological study of HBV, HCV and HTLV-1 in Sherpas of Nepal. Asian Pac J Cancer Prev. 2004;5:370-3.

83. Shah B, Bhattacharya S, Parija S. Seroprevalence of hepatitis B virus among Bhutanese refugees residing in Nepal. Kathmandu Univ Med J. 2005;3(3):239-42.

84. Shrestha P, Bhandari D, Sharma D, Bhandari B. A study of viral hepatitis during pregnancy in Nepal Medical College Teaching Hospital. Nepal Med Coll J. 2009;11(3):192-4.

85. HEPA Foundation, United Nations Office on Drugs and Crime (UNODC). Prevalence of hepatitis C in OST client.

86. Asian Network of People Who Use Drugs. Barriers to hepatitis C diagnosis, management and treatment among people who inject drugs in 4 Asian countries: a community led study in India, Indonesia, Malaysia \& Nepal. Bangkok: Asian Network of People who Use Drugs (ANPUD); 2011.

87. Adhikari P, Pathak U, Uprety D, Sapkota S. Profile of ascites patients admitted in Nepal Medical College Teaching Hospital. Nepal Med Coll J. 2012;14(2):111-3.

88. Ojha CR, Khagendra K, Shakya G. Co-infection of hepatitis C among HIVinfected population with different risk groups in Kathmandu, Nepal. Biomed Res. 2013;24(4):441-4.

89. Supram HS, Gokhale S, Sathian B, Bhatta DR. Hepatitis B virus (HBV) and hepatitis $C$ virus (HCV) co-infection among HIV infected individuals at tertiary care hospital in Western Nepal. Nepal J Epidemiol. 2015;5(2):488-93.

90. Ministry of Health and Population. National Centre for AIDS and STD Control. Integrated biological and behavioral surveillance (IBBS) survey among people who inject drugs (PWID-male) in the Eastern Terai Highway Districts (Jhapa, Morang and Sunsari) of Nepal, 2015. Teku, Kathmandu: Government of Nepal; 2015.

91. Ministry of Health and Population. National Centre for AIDS and STD Control. Integrated biological and behavioral surveillance (IBBS) survey among people who inject drugs (PWID) in Kathmandu Valley, 2015. Teku, Kathmandu: Government of Nepal; 2015.

92. Ministry of Health and Population. National Centre for AIDS and STD Control. Integrated biological and behavioral surveillance (IBBS) survey among people who inject drugs (PWIDs) in Pokhara Valley, 2015. Kathmandu: Government of Nepal; 2015.

93. United Nations Development Program/Department for International Development/Center for Molecular Dynamics Nepal. Draft report of four PLHIV surveys. 2012

94. Loewinger G, Sharma B, Karki DK, Khatiwoda P, Kainee S, Poudel K. Low knowledge and perceived hepatitis $C$ risk despite high risk behaviour among injection drug users in Kathmandu, Nepal. Int J Drug Policy. 2016;33:75-82.

95. Ministry of Health. National Centre for AIDS and STD Control. Integrated biological and behavioral surveillance (IBBS) survey among female injecting drug users in Kathmandu Valley. Teku, Kathmandu: Government of Nepal; 2016.

96. Ministry of Health. National Centre for AIDS and STD Control. Integrated biological and behavioral surveillance (IBBS) survey among people who inject drugs (PWID-male) in Western to Far Western Terai Highway Districts of Nepal. Kathmandu: Government of Nepal; 2016

97. Nepal A, Kunwar B. Evidence of hepatitis C virus infection and associated treatment in Nepal. J Mol Biomark Diagn. 2016:7(2):270. 